\title{
Brownian motion: a paradigm of soft matter and biological physics
}

\author{
Erwin Frey ${ }^{1,3},{ }^{*}$ and Klaus Kroy ${ }^{2,3},{ }^{* *}$ \\ ${ }^{1}$ Arnold Sommerfeld Center and CeNS, Department of Physics, Ludwig-Maximilians-Universität \\ München, Theresienstrasse 37, 80333 München, Germany \\ ${ }^{2}$ Institut für Theoretische Physik, Universität Leipzig, Linnestr. 5, 04103 Leipzig, Germany \\ ${ }^{3}$ Hahn-Meitner Institut, Glienicker Strasse 100, 14109 Berlin, Germany
}

\begin{abstract}
Key words Brownian motion, fluctuations, biological physics, stochastic processes.
PACS 05.40.-a, 82.39.-k, 87.15.-v

This is a pedagogical introduction to Brownian motion on the occasion of the 100th anniversary of Einstein's 1905 paper on the subject. After briefly reviewing Einstein's work in its contemporary context, we pursue some lines of further developments and applications in soft condensed matter and biology. Over the last century Brownian motion became promoted from an odd curiosity of marginal scientific interest to a guiding theme pervading all of the modern (live) sciences.
\end{abstract}

\section{Introduction: 100 years ago}

"If, in some cataclysm, all of scientific knowledge were to be destroyed, and only one sentence passed on to the next generation of creatures, what statement would contain the most information in the fewest words? I believe it is [...] that all things are made of atoms - little particles that move around in perpetual motion, attracting each other when they are a little distance apart, but repelling upon being squeezed into one another." Some decisive theoretical tools to put the atomic hypothesis (here quoted from The Feynman Lectures [1]) at scrutiny were provided in Einstein's paper “Über die von der molekulartheoretischen Theorie der Wärme geforderte Bewegung von in ruhenden Flüssigkeiten suspendierten Teilchen" [2] published in this journal 100 years ago, which starts with the claim "that according to the molecular-kinetic theory of heat, bodies of a microscopically visible size suspended in liquids must, as a result of thermal molecular motions, perform motions of such magnitudes that they can be easily observed with a microscope." It quickly became clear that Einstein's calculations could explain hitherto mysterious observations of dancing pollen granules and other small particles first systematically described by the Scottish botanist Robert Brown [3] and (independently) by his French colleague Adolphe Brongniart [4]. And a few years later, drawing upon painstaking observations of thousands of Brownian particles in his lab at the Sorbonne, Jean Perrin could ultimately end the debate about the reality of atoms [5]. Though dating back to the ancient Greeks, this debate had considerably intensified towards the turn of the century and prompted Planck's dictum that the acceptance of new scientific ideas proceeds via natural extinction rather than by persuasion of the opponents, which is sometimes referred to as Planck's principle among philosophers of science.

It was soon realized that the laws of Brownian motion do not exclusively pertain to the realm of physics but likewise to chemistry, biology and even economics. After the dust had settled, this was acknowledged by a couple of Nobel prizes: in physics, Perrin was rewarded for proving the reality of atoms while the

* Corresponding author E-mail: frey@1mu.de

** E-mail: kroy@hmi.de 
chemists at the same time honored Svedberg for his dispersion studies and for the invention of the ultracentrifuge, and later Onsager for further growing the germ layed in Einstein's 1905 paper. Also, the economists eventually embraced the laws of Brownian motion as useful tool for the valuation of stock options in due time for the centenary of their derivation by Bachelier [6]. Since he (and other contributors) had unfortunately passed away by that time, the prize went to Merton and Scholes ${ }^{1}$.

From the historical perspective, the theory brought forward in Ref. [2] (together with related work by Smoluchowski [8] and Langevin [9]) is therefore certainly not to be sniffed at by her two sisters, relativity and quantum mechanics. It took quite a different course, though. While the latter two were soon promoted to the holy grail of science and have never since ceased to attract the public, the former catched much less attention. This is all the more astonishing as the vision it promoted, namely that our macroscopic world including its living organisms resides on a jittering sea of fluctuations, is particularly remarkable for the broadness of its impact. During the last 100 years, the facets of this vision have continuously multiplied, thereby providing ample evidence for a "slower" [10], yet "unfinished" [11] revolution. Brownian motion remains a leading paradigm in a development that seems directed oppositely to that of the quantum $\&$ relativity revolution - not deeper into the elements but towards the understanding of the principles underlying the emergence of collective properties in many-particle assemblies ${ }^{2}$ - and for which P. W. Anderson has coined the mantra "more is different" [14], meaning that "at each new level of complexity, entirely new properties appear, and the understanding of these behaviors requires research which I think is as fundamental in its nature as any other." Depending on the importance of thermal fluctuations one may roughly divide this research into "hard matter" (" $k_{B} T \rightarrow 0$ ") and "soft matter" $[15,16]\left(" k_{B} T \simeq 1\right.$ ") physics. Underlying both is the discipline of statistical mechanics, which has not only become a major part of any physics curriculum, but which has over the years proven its vitality by invading numerous foreign disciplines such as biology, ecology, economics, traffic, sociology, game theory, etc. [19-24], and by identifying new interdisciplinary research topics like criticality, structure formation and synergetics, quenched disorder, glassy dynamics, and many others, where it tries to bring to bear its concepts and methods. On the other hand, widespread hopes that on a more fundamental, sub-atomic scale fluctuations would eventually cease and give way to solidity and firm order did not quite materialize. Instead, the toolbox of statistical mechanics turned out to be instrumental also to various "fundamental" developments from quantum theory to cosmology (path integrals, stochastic quantization, space-time foam, ...).

In the following, our scope will have to be much, much narrower, though, and we will try to restrict ourselves to tracing a selective set of implications and modern offsprings of Einstein's 1905 paper on Brownian motion [2], with the aim of illustrating variations of its basic themes in soft condensed matter and biological physics. Since, above all, Brownian motion is particularly strongly linked to biology, not only by its name; a link that we welcome as a guide (or excuse) for taking shortcuts through the bewildering jungle of applications. Soft matter and biological physics are both fields governed by meso-scale structures for which thermal fluctuations are all important, the relevant dynamics often bridging the gap between the microscopically fast and the macroscopically slow, as neatly summarized by the formulas

$$
k_{B} T \approx 4 \mathrm{pN} \cdot \mathrm{nm}, \quad k_{B} T / \eta_{\mathrm{w}} \approx 4 \mu \mathrm{m}^{3} \mathrm{~s}^{-1},
$$

where $\eta_{\mathrm{w}}$ denotes the viscosity of water. Conversely, these formulas immediately raise the (legitimate) expectation that Brownian motion is an ubiquitous phenomenon in these fields.

More precisely, our outline is as follows. After a brief historical introduction to Einstein's paper [2] and closely related work in section 2 , the remainder is organized along six traces. Our starting point is the observation that the derivation in Ref. [2] inspired and partly anticipated major developments in

\footnotetext{
1 Commonly better known for their role in one of the world's largest financial collapses [7].

2 In view of beautiful recent progress in what one might call "condensed matter cosmology" (e. g. [12, 13]), this antithesis seems less compelling now than some years ago.

3 Here, the characteristic intrinsic energy scale of the system is understood to provide the unit. In this sense, $k_{B} T \approx 1$ may for instance indicate that Brownian motion limits our auditory abilities [17], or the accuracy of a gravitational wave detector [18] or (in the form of Nyquist-Johnson noise) of any electrical measuring instrument.
} 
non-equilibium statistical mechanics (section 3) as well as a powerful stochastic calculus (section 4) with innumerable applications, of which we only mention a few paradigmatic examples taken from biology in section 5. Precisely as proposed by Einstein in 1905 [2], Brownian particles have ever since served as versatile probes of their environment (section 6). Moreover, their stochastic fluctuations have provided valuable insights into their own (soft) internal degrees of freedom, which can give rise to most complex macroscopic material properties thus providing a unique potential for applications (section 7). Finally, Brownian fluctuations are both exploited and rectified in ingenious biological micro-machines (section 8).

Needless to say that, even within this limited scope, we cannot be objective or complete in any sense. Original publications can by no means be cited systematically, and citations should rather be taken as our (certainly biased) personal suggestions for further reading. With these cautious remarks, we hope that the reader is prepared for a very subjective random walk lurching to and fro between physics and biology.

\section{Historical notes: from Brown to Perrin}

\section{Brownian motion: a very brief history}

"The story of Brownian motion is one of confused experiment, heated philosophy, belated theory, and, finally, precise and decisive measurement" [25]. It is well documented in various publications by Perrin, Nye, Brush, and others, and summarized in several monographs on Brownian motion (e. g. [26-29]). Here, we content ourselves with a few remarks. First, since we expect 2005 to be a year with strong "Matthew effects" (in case of doubt, credit is ascribed to those who already got most [30]), it seems in order to start by a brief disclaimer: Brown was not the first observer of Brownian motion (similar observations had repeatedly been made after the first microscopes came up in the late 17th century); Perrin was not the first to show that kinetic theory is in accord with experiments, nor was the admirable diligence of his group necessary to make the atomic hypothesis acceptable to the majority of researchers; Einstein did of course not invent the diffusion equation - which he supposed to be generally known just as we would today nor did he first suggest that Brownian motion is due to thermal motion of the solvent molecules or provide the first testable prediction of statistical mechanics or kinetic theory, etc.

In fact, Brown was curious enough about the erratic dance under the microscope to spend some time in the late 1820 's on systmatic investigations, trying to clarify its origin. Starting out with micrometersized granules from the pollen of Clarkia pulchella and later using several other substances, he convinced himself (though not everybody else) that internal animate as well as external perturbative causes and optical illusions could be ruled out. Nevertheless, the phenomenon hardly received the attention it deserved. It remained a curiosity during Brown's lifetime and almost until the end of the century, when Léon Gouy's detailed investigations of the phenomenon [31] led him to the conclusion that the ubiquitous perpetual motion is caused by thermal motion of the solvent molecules. (This was in 1888, when Einstein still went to primary school, and even at that time the idea was not really new ${ }^{4}$ ) He later also noticed that this explanation seemed to be in contradiction to the second law of thermodynamics (a concern independently raised by Röntgen in a letter to Einstein in 1906) or could at least provide an ideal natural laboratory for direct experimental examination of certain apparent logical contradictions between the atomic hypothesis and thermodynamics. By that time diffusion was a well-established thermodynamic notion routed in the work "Über Diffusion" (1855) by the German pathologist Adolf Fick, which starts with the words "Hydrodiffusion through membranes is not only one of the basic factors of organic life ${ }^{5}$, but also a most interesting physical process that deserves much more attention from physicists than it has got so far" [34]. But the relation between Brownian motion and diffusion — today often regarded as synonymous — was

${ }^{4}$ See e. g. C. Wiener, "Erklärung des atomistischen Wesens des tropfbar-flüssigen Körperzustandes und Bestätigung desselben durch die sogenannten Molecularbewegungen" [32].

5 Some advocates of the opposite view nowadays claim to have compelling evidence that "there is certainly no reason to suppose that classical diffusion theory, or any of its offspring, will play a significant role in our understanding of biology in the future". [33] 
much less clear. This was the missing link provided by Einstein and Smoluchowski, who thereby pointed the way to quantitative hands-on experiences with a strange microcosmos awaiting discovery. Despite of work by Clausius, Maxwell, Boltzmann, Loschmidt and others on heat conduction and interdiffusion that had clearly demonstrated the usefulness of kinetic theory and even produced reasonably accurate estimates for the size of molecules by the late 19th century, there remained strong opposition against taking the underlying atomistic view too literal. Only Perrin's extensive studies, performed with Einstein's prediction at hand, could finally convince the last of the "Energetiker" around Ostwald that atoms were more than just a convenient analogy. To us, the great impact of Perrin's and Einstein's work on Brownian motion seems partly linked to the fact that, instead of only measuring another thermodynamic or hydrodynamic relation that could be rationalized by kinetic theory, a forthright manifestation of molecular chaos, namely the predicted "strange" equation of motion of individual diffusing colloids (on average the travelled distance grows with the square root of time), could directly be observed. The fractal trajectory implied by Einstein's result provided a convincing explanation why earlier attempts to measure the velocity of Brownian particles and compare it to the average thermal velocity predicted by kinetic theory (for free particles), were in vain: with the characteristic distance travelled by the particle growing as $\sqrt{t}$, the velocity is proportional to $1 / \sqrt{t}$. Naturally, around 1900, a non-differentiable trajectory was hard to swallow, and Einstein hastened to add [35] that the molecular discreteness provides a lower cutoff to such unseemliness. Curiously, the same fractal trajectories were determined to reappear later in the disguise of the Heisenberg uncertainty relation, which indicated that they also prevail on a more fundamental level [36] (see section 4).

Finally, it should be said that Einstein's interest in Brownian motion grew out of a profound interest in statistical fluctuations [35,37,38], which can be identified as a common denominator of his work in seemingly unrelated areas. In particular, fluctuations played a crucial role [39] in his persistent efforts to find a rational explanation for Planck's radiation law. Einstein realized that stochastic fluctuations rule on the quantum level (photon statistics) in classical small systems (Brownian motion) and near a critical point (critical opalescence), thereby anticipating at the beginning of the 20th century, what became vast fields of research towards its end. Without exaggerating, he may therefore be called the father of fluctuation theory [40].

\section{Revision course: Einstein's 1905 paper on Brownian motion}

In brief, the argument in Ref. [2] goes as follows. If the atomic hypothesis holds, particles immersed in an ambient medium must undergo perpetual irregular motion due to chaotic collisions with its molecules. It was Einstein's insight (cited above) that micrometer-sized particles are both big enough to be seen themselves in the microscope and small enough that their Brownian motion is substantial, and, as a consequence, keeps them suspended indefinitely against gravity. The publication consists of two major parts. In the first part, Einstein argues against the view — still common at that time - that suspended particles may be dismissed in thermodynamics. He insists that $N$ particles of arbitrary volume $v$ suspended in a solvent at small number density $n \ll v^{-1}$ represent an ideal gas and therefore give rise to an osmotic pressure $p=n k_{B} T$ (in modern notation), thereby excerting a finite, albeit small, thermodynamic force on their container. In presence of a constant volume force $\boldsymbol{K}$ acting on the particles one thus has the (isothermal) force balance $n(\boldsymbol{r}) \boldsymbol{K}+\boldsymbol{\nabla} p(\boldsymbol{r})=0$, or $p(\boldsymbol{r})=k_{B} T n(\boldsymbol{r}) \propto e^{-\boldsymbol{K} \cdot \boldsymbol{r} / k_{B} T}$ in the steady state. Further, Einstein suggests to regard this stationary balance as a dynamic equilibrium between a diffusion current $-D \nabla n$ and a drift current $n \boldsymbol{K} / \zeta$, with $\zeta$ and $D$ the friction and diffusion coefficient of the Brownian particles in the solvent, respectively. Eliminating $K$ and $n$ between the balance of forces and that of drift and diffusion, he infers what is today known as Stokes-Einstein relation

$$
D=k_{B} T / \zeta
$$


So far, this is a relation between two coefficients appearing in two generalized hydrodynamic equations, namely the Stokes equation for incompressible viscous flow ${ }^{6}$, and the diffusion equation. This is not yet quite, what made it so remarkable. We must point out that the notion of Boltzmann's constant $k_{B}$ was not yet fully established at the time, and its apparence in Eq. (2), originally in the form of the gas constant divided by Avogadro's number, allowed the latter to be determined from measuring macroscopic quantities. Intriguingly, it thereby indicated a relation of the macroscopic kinetic coefficients $D, \zeta$ to the microscopic, molecular world.

This link, which was later formalized under the name of Green-Kubo relations, is established in the second part of Einstein's paper, which is quoted as a pedagogical introduction to stochastic processes in Gardiner's textbook [28]. It undertakes a probabilistic derivation of the diffusion equation based on a particularly simple model for the fluctuations of the Brownian particle, called a random walk. The essential idea is to express the particle concentration (in one space dimension) at position $x$ and time $t+\tau$ as a function of the concentration at time $t$,

$$
n(x, t+\tau)=\int \mathrm{d} \xi n(x+\xi, t) \varphi_{\tau}(\xi)
$$

Due to the use of the auxiliary "jump propabilities" $\varphi_{\tau}(\xi)$ in this Chapman-Kolmogorov equation, as it is called today, Einstein's derivation appears much more elegant and transparent than Smoluchowski's more mechanistic discussion of Brownian motion, because it circumvents the construction of an explicit microscopic dynamical theory of the process by some (implicit) technical assumptions on $\varphi_{\tau}(\xi)$. Taylor expanding $n(x, t)$ with respect to the "small" quantities $\tau$ and $\xi$ and matching the leading order terms on both sides, Einstein recovers the diffusion equation

$$
\partial_{t} n(\boldsymbol{r}, t)=D \nabla^{2} n(\boldsymbol{r}, t)
$$

along with a stochastic expression for the diffusion coefficient,

$$
2 D=\frac{1}{\tau} \int \mathrm{d} \xi \xi^{2} \varphi_{\tau}(\xi)=\frac{\left\langle\delta x^{2}\right\rangle}{\tau}=\frac{\left\langle\delta \boldsymbol{r}^{2}\right\rangle}{3 \tau} .
$$

The expressions for $D$ are understood to be independent of the considered time interval $\tau$ as long as $\tau$ is large compared to a microscopic collision time. In his derivation Einstein also invokes the limit " $\tau \rightarrow 0$ ", which later became the subject of debate (see below). The final relation between $D$ and the mean square displacement $\left\langle\delta \boldsymbol{r}^{2}(t)\right\rangle \equiv\left\langle[\boldsymbol{r}(t)-\boldsymbol{r}(0)]^{2}\right\rangle$ of a Brownian particle starting at $t=0$ at $\boldsymbol{r}=\boldsymbol{r}(0)$ follows from applying the diffusion equation to each of the diluted particles separately. It is of interest as paradigm for a general rule: kinetic coefficients (here $D, \zeta$ ) in macroscopic thermodynamic or hydrodynamic equations (here diffusion, Stokes equation) can entirely be expressed in terms of correlation functions of fluctuations of microscopic variables (see section 3). This is how the discrete chaotic processes on the microscale generate the smooth behavior on the macroscopic scale.

\section{Non-equilibrium statistical mechanics: from Stokes-Einstein relations to fluctuation theorems and effective temperatures}

\section{Close to equilibrium: the fluctuation-dissipation connection}

The most celebrated result of Eintein's 1905 paper on Brownian motion [2] is the so-called Stokes-Einstein relation that links two hydrodynamic transport coefficients to each other and to thermal fluctuations, see

\footnotetext{
6 This is nothing but the diffusion equation, Eq. (4), with $D$ replaced by the kinematic viscosity, and $n(\boldsymbol{r})$ replaced by the solvent velocity field projected onto its "transverse" components (so that it is non-divergent).
} 
Eqs. (2), (5). The second part of it, the relation between hydrodynamic transport coefficients and microscopic fluctuations, is today more commonly known as Green-Kubo relation, and is usually presented in the form [41]

$$
D=\frac{1}{3} \int_{0}^{\infty} \mathrm{d} t\langle\boldsymbol{v}(t) \cdot \boldsymbol{v}(0)\rangle .
$$

As Einstein himself noticed [35,37], the content of Eq. (6) is not restricted to Brownian motion but is straightforwardly translated to electrical circuits ${ }^{7}$, a case that was later further elaborated by Johnson and Nyquist $[43,44]$. Analogous relations have been derived for all kinds of transport coefficients (conductivities, viscosities, etc.) relevant in different areas of condensed matter physics. They are of considerable practical interest, as they suggest a way to do non-invasive measurements of response coefficients (here $\zeta)$. And they provide insight into the complicated behavior of strongly interacting many-particle systems. For example, the general form of Eq. (6) persists for dense suspensions of Brownian particles, where the gradient diffusion coefficient governing the diffusive spread of concentation variations via the diffusion equation becomes renormalized by the (osmotic) compressibility of the suspension compared to the bare diffusion coefficient of a single particle in the solvent. This knowledge allows one (among other things) to infer why the dynamics slows down near phase transitions, where susceptibilities (here the compressibility) diverge. Thus, near a critical point [45], fluctuations not only become long-ranged as foreseen by Einstein in his paper on critical opalescence [38], but they also become long-lived; though in a slightly more intricate way than suggested here $[46,47]$.

It was Onsager who started the excavation of the treasure buried in the Stokes-Einstein relation. Following Einstein's later work [38] on fluctuations, he likened the relaxation of hydrodynamic variables in response to weak external perturbations to the decay of correlations between the corresponding microscopic variables: the forced deviation from equilibrium could as well have been a random fluctuation of the system. This regression principle allowed him to exploit exact microscopic symmetries, notably the invariance of the microscopic equations of motion under time reversal, to infer constraints on the kinetic coefficients: however difficult and incomprehensible e. g. the coefficient of thermodiffusion (Soret effect) ${ }^{8}$ may seem, thanks to Onsager we can exactly relate it to a corresponding coefficient for "diffusive heating" (Dufour effect). Further generalizations of Eq. (2) like the mentioned Green-Kubo relations and the fluctuationdissipation theorem followed. Around these cornerstones the field of linear non-equilibrium thermodynamics (also "irreversible thermodynamics", "generalized/molecular hydrodynamics") developed. The modern derivation [49-51] of all of the mentioned relations is based on linear-response theory (often complemented by projection operator techniques [52] to separate slow, hydrodynamic from fast, fluctuating variables), which therefore can be regarded as the foundation of "close-to-equilibrium statistical mechanics"; see Kubo's short pedagogical overview [41]. The basic assumption underlying linear-response theory is that the phase-space density may be linearized in a small perturbation added to the Hamiltonian of the system. This has been the subject of some debate, reviewed in [53]: since single trajectories of nonlinear dynamical systems are known to exhibit sensitive dependence on perturbations, the required macroscopic linearity cannot be a property of single trajectories but rather has to rely on some ergodic property of the phase space dynamics as a whole [54]. Only for toy models such as the periodic Lorentz gas or Sinai billiard [55], the link between the transport coefficients and the underlying microscopic Hamiltonian dynamics can be made fully explicit $[53,56]$, while for the complex systems generally of interest in statistical mechanics this is out of reach. Already a rigorous microscopic derivation of Fourier's law for heat conduction, which is a diffusion equation with $D$ replaced by the thermal conductivity, turns out to be quite an endeavor (most recently reviewed in Ref. [57]). Similarly, it was recently disputed (see

\footnotetext{
7 Since he could not imagine other testable consequences, he dismissed any discussion of further realizations as "useless" [35] but maintained interest in the electrical analog [37], for which he even designed a "Maschinchen" (little machine) [42] to do experiments by himself.

${ }^{8}$ See e. g. Ref. [48] for a recent experimentally oriented review, and various contributions in Eur. Phys. J. E 15(1), 2004.
} 
[58]) whether extremely careful measurements [59] might be able to establish the role played by chaotic microscopic dynamics for Brownian motion. So the question, whether chaotic microscopic dynamics or the high dimensionality of the phase space is responsible for the irregularity of Brownian motion is, after a century, still open for investigation.

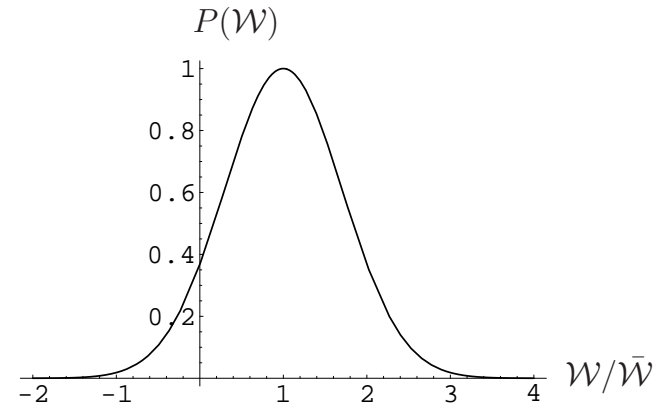

Fig. 1 Probability distribution for the dissipative work $\mathcal{W}$. The finite weight for negative $\mathcal{W}$ implies that Einstein's theory of Gaussian fluctuations, if applied to $\mathcal{W}$, predicts transient violations of the second law.

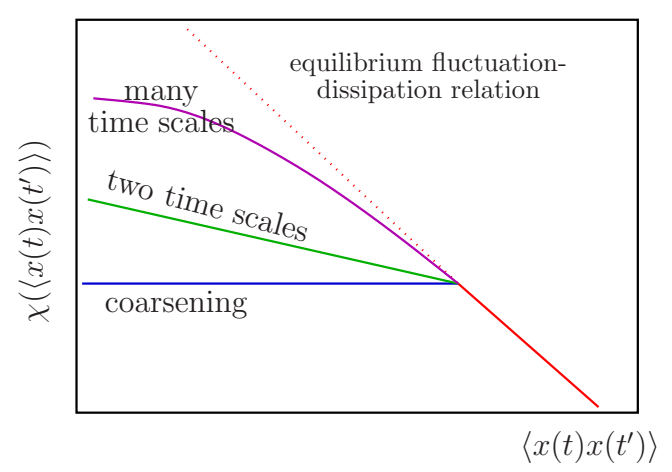

Fig. 2 Various scenarios for generalized fluctuationdissipation relations represented in integrated form: $\chi=\int_{t^{\prime}}^{t} d \tau \partial\langle x(t)\rangle / \partial F\left(t^{\prime}\right)$

\section{Far from equilibrium: "violating" basic laws and aging}

Far from equilibrium, the whole formalism of irreversible thermodynamics is a priori not generally applicable. However, it is a natural temptation to try to extend the approved formalism to classes of problems that are in some generic sense far away from equilibrium, such as driven diffusive systems [60], sheared fluids [61] or gelled [62] suspensions of Brownian particles, a living cell [63] or RNA molecules or protein domains unfolding upon application of an external pulling force [64], etc. Can, for such systems, still general statements be made that save us from having to solve the full dynamics? One approach tries to extend the classification scheme of universality classes [46] based on the renormalization group and equilibrium critical points to systems far from equilibrium [65] (see section 4). Closer related to the topics so far dealt with in the present section are two interesting lines of investigation that try to generalize the fluctuation dissipation theorem, which we recall is a generalization of the Stokes-Einstein and Green-Kubo relations. Both are to date still controversial and the subject of heated debates (e. g. [66]).

The first is concerned with the intensely discussed paradigm of glassy dynamics [67]. Glassy dynamics is characteristic not only of structural glasses (such as colloidal, polymeric, molecular, or metallic glasses) and spin glasses but of a much wider class including many soft [68], disordered [69], granular [70], and in particular biological (e. g. proteins [71]) systems. As generic explanation for the origin of glassy dynamics several tentative mechanisms have been proposed (purely kinetic models, drop models, trap models, freeenergy landscapes, etc. [68,72-74]), and their further exploration to unveil the intricate roles played by disorder and fluctuations remains a formidable challenge in theoretical physics. In contrast to the dynamics in supercooled liquids that approach the glass transition from the liquid side, which is rather well understood (see our discussion towards the end of section 6), the emphasis here is on the non-equilibrium dynamics after falling out of equilibrium, which is characterized by aging (i. e. relaxing indefinitely, in general in a non-universal manner, without ever reaching equilibrium) [73]. Yet, a generalized fluctuation-dissipation theorem $\left(t \geq t^{\prime}\right)$

$$
\frac{\delta\langle x(t)\rangle}{\delta F\left(t^{\prime}\right)}=\frac{1}{k_{B} \mathcal{T}\left(t^{\prime}\right)} \frac{\partial}{\partial t^{\prime}}\left\langle x(t) x\left(t^{\prime}\right)\right\rangle
$$


may still hold [73] (see figure 2). Close to equilibrium, $\mathcal{T}$ is the ordinary ambient temperature $T$ and both sides depend on the time difference $t-t^{\prime}$ only. Equation (7) is then just a time-dependent generalization of Eqs. (2), (5) and the Stokes law $F=\zeta v$ : the ordinary equilibrium fluctuation-dissipation theorem. Far from equilibrium (e. g. below the glass transition temperature), this is still the case for $t-t^{\prime} \ll t^{\prime}$, where $t^{\prime}$ is identified with the waiting time after falling out of equilibrium. For $t-t^{\prime} \gg t^{\prime}$ time-translation invariance and the relation between $\mathcal{T}$ and the ambient temperature are lost. The response and fluctuation functions in Eq. (7) are then dominated by their aging parts. The hope is that the effective temperature $\mathcal{T}\left(t^{\prime}\right)$ will still exist and be independent of the variable (here $x$ ) and consistent with alternative definitions of effective temperatures that are a priori independent of Eq. (7) ${ }^{9}$. This, in fact, would justify the notion of a generalized or quasi fluctuation-dissipation theorem [75]. While the theoretical understanding of these issues emerged from mean-field spin glass models [76], a corresponding development for structural glasses (e. g. suspensions of Brownian particles) is still at its beginning [77].

As a second line of attack towards the realm far from equilibrium, we want to mention the so-called non-equilibrium work relations or fluctuation theorems. These are theorems supposed to remain valid beyond linear response, far from equilibrium, and in particular also for small systems ${ }^{10}$, away from the thermodynamic limit, where transient violations of the second law of thermodynamics become increasingly likely. The fluctuation theorems precisely say how likely. The recent excitement about these developments was initiated by the chaotic hypothesis of Gallavotti and Cohen [79] and independent work by Jarzynski [80], and it has even been suggested that non-equilibrium statistical mechanics is presently undergoing a foundational period comparable to that in equilibrium statistical mechanics a century ago [81]. The field has last been reviewed in Refs. [82-86]. Here, we try to sketch the basic idea. Consider a system prepared in thermal equilibrium with its environment at temperature $T$ (e. g. an RNA molecule). The system shall be repeatedly subjected to an identical perturbation protocol such that the external work $W$ is performed on it. In contrast to the reversible work $W_{0}$, which is a state function, $W$ fluctuates from one repetition to the next. If the average over the (infinitely many) realizations of this process is denoted by an overbar and the dissipative work by $\mathcal{W} \equiv W-W_{0}$, Jarzynski's equality may be written in the form [85]

$$
\overline{e^{\mathcal{W} / k_{B} T}}=1
$$

Obviously, the dissipative work must sometimes be positive as well as negative; i. e. sometimes mechanical work will be gained from the heat bath (see figure 1). This is indeed what Einstein's theory of fluctuations [38] requires. Already if the protocol does not take the system too far from equilibrium, so that fluctuations of $\mathcal{W}$ can be assumed to be Gaussian distributed about an average dissipation $\overline{\mathcal{W}}$, there will be some finite weight in the tail with negative $\mathcal{W}$. This is not only consistent with the second law of thermodynamics, the latter is in fact immediately deduced from Eq. (8) via Jensen's inequality $\left(\overline{e^{x}} \geq e^{\bar{x}}\right)$. Equation (8) can be used to infer the reversible work $W_{0}$ from many repeated measurements of the irreversible work $W$. This has been tested experimentally, e. g. for a Brownian particle dragged through water [87, 88], and for RNA [64] and a number of other biomolecules subjected to mechanical forces (see Ref. [86] for an overview and suggestions for further experiments).

\section{Stochastic processes: the universal toolbox}

\section{Universality: the reign of large numbers}

In section 2 we praised the elegance of Einstein's derivation compared to Smoluchowski's attempt to construct a microscopic dynamical theory of Brownian motion. This is not merely a matter of elegance,

\footnotetext{
${ }^{9}$ The effective temperature may, however, exhibit a more complicated time dependence of the form $\mathcal{T}\left(\left\langle x(t) x\left(t^{\prime}\right)\right\rangle\right)$, as observed in certain spin glasses, or simply diverge (corresponding to a universal "trivial" aging dynamics), as e. g. in ordinary coarsening during an equilibrium phase transition.

${ }^{10}$ Generally, care needs to be taken that the notion of temperature is still valid, which can be problematic for small systems [78].
} 
though. While the strength of Smoluchowski's approach lies in its ability to give insights into the microscopic mechanism of Brownian motion, Einstein's approach relied on the idea that certain aspects ${ }^{11}$ of $^{-}$ fluctuations are independent of the details of the underlying microscopic dynamic processes, as long as they are sufficiently many and sufficiently uncorrelated in time and space (both aspects also played a role in section 3). If this is so, can one develop a universally applicable calculus of stochastic phenomena? Indeed, this is essentially what the theory of stochastic processes aims at.

Einstein's derivation utilized the Chapman-Kolmogorov equation Eq. (3) that translates the rules of a postulated simplistic time-discrete jump pr ocess of individual Brownian particles encoded in the jump propabilities $\varphi_{\tau}(\xi)$, to the particle concentration $n(x, t)$. Equivalently he could also have started from a formulation that is discretized in space and continuous in time and write down a master equation

$$
\frac{d}{d t} P(x, t)=\sum_{\xi}\left[w_{\xi \rightarrow x} P(\xi, t)-w_{x \rightarrow \xi} P(x, t)\right]
$$

for a single particle with probabilities $P(x, t) \equiv n(x, t) / N$ and jump rates $w_{\xi \rightarrow x}$. Upon taking the limit of a large number of independent jumps, one obtains along either way a coarse-grained description in form of the diffusion equation Eq. (4). It is important to note that in taking this limit, some information about the underlying stochastic process, e. g. about the trajectory $x(t)$, is irretrievably lost. The ChapmanKolmogorov equation Eq. (3) and the master equation Eq. (9) provide a more detailed or microscopic description than the diffusion equation, Eq. (4). Indeed, Einstein exploits this fact by implicitely assuming that the difference between the actual microscopic dynamics, which Smoluchowski struggled to calculate, and the simplistic random walk will not matter for the end results Eqs. (4), (5). The idea becomes maybe most transparent from yet another equivalent approach Einstein could have taken. Starting from the discrete trajectory

$$
x(t)=\sum_{n=1}^{N} \xi(n \tau)
$$

of a single particle with independent and identically distributed random jump lengths $\xi$, he could have appealed to the law of large numbers. Provided the first two moments of the jump distribution $\varphi_{\tau}(\xi)$ exist, the cental limit theorem guarantees that the limiting probability density for the individual particle trajectories $x(t)$ starting at $t=0$ at the origin is given by the Gaussian [91]

$$
P(x, t)=\frac{1}{\sqrt{4 \pi D t}} \exp \left[-\frac{x^{2}}{4 D t}\right] \text {. }
$$

For the independent Brownian particles Einstein considered, this statement is of course equivalent to saying that the particle concentration $P(x, t)$ obeys the diffusion equation, Eq. (4). In fact, Eq. (11) is nothing but the Green function of Eq. (4), i. e. its solution to the initial condition $P(x, 0)=\delta(x)$.

Given such a powerful attractor of probability distributions, it is an intriguing question, whether there is a particularly simple member of the class of all stochastic processes with probability densities that converge to Eq. (11). Is there a simplest representative trajectory corresponding to the diffusion equation? This question was positively answered by Wiener in 1921 [92]. Inserting Eq. (11) with a short time interval $t=\tau$ for the jump probabilities $\varphi_{\tau}(\xi)$ in the Chapman-Kolmogorov equation Eq. (3) and iterating the latter one arrives at the expression

$$
P(x, t)=\int \prod_{j=1}^{N-1} \frac{d x_{j}}{\sqrt{4 \pi D \tau}} e^{-\sum_{j=0}^{N-1} \frac{\left(x_{j+1}-x_{j}\right)^{2}}{4 D \tau}} \rightarrow \int_{x(0)=0}^{x(t)=x} \mathcal{D}[x(\tau)] e^{-\frac{1}{2 D} \int_{0}^{t} \frac{1}{2}\left(\frac{\partial x}{\partial \tau}\right)^{2} d \tau} .
$$

\footnotetext{
11 Precisely which aspects, is currently still an intriguing open question (see e. g. [89, 90]).
} 
The final path-integral representation is obtained upon taking the continuum limit $N \rightarrow \infty$ with $N \tau=t$, and has a well-defined meaning within the framework of measure theory [92]. Wiener could also characterize the typical paths corresponding to the limit taken in Eq. (12) and show that they are unphysical fractals, continuous but non-differentiable curves (see Fig. 4). One can imagine them to be constructed by a self-similar continuation of the large-scale structure of a trajectory of a Brownian particle to arbitrarily short distances. The intricate details of the actual physical process at short scales together with the corresponding characteristic microscopic length scale are simply wiped off to give way to the ultimate simplicity of ideal self-similarity. The innocent looking prescription given after Eq. (12) actually encodes a renormalization of the original discrete stochastic model to a mathematically more convenient scale-free phenomenological model, known as the Wiener process.

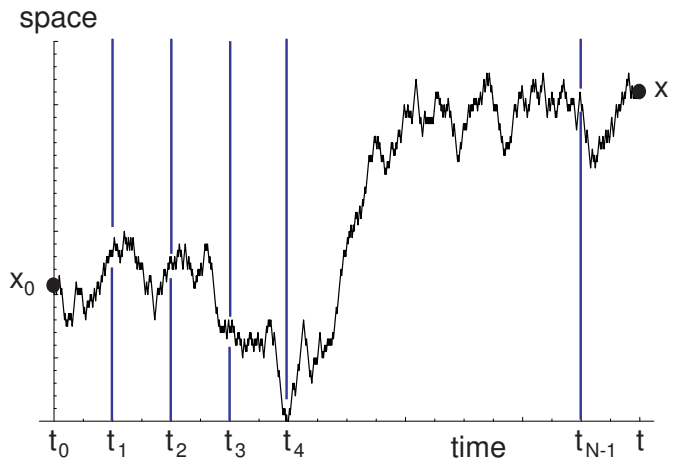

Fig. 3 Illustration of the concept of a path integral: trajectory of a Brownian particle starting at position $x_{0}$ at time $t_{0}$ and passing through $N$ gates of size $d x_{1}, d x_{2}, \cdots, d x_{N-1}, d x$ at times $t_{1}, t_{2}, \cdots, t_{N-1}, t$.

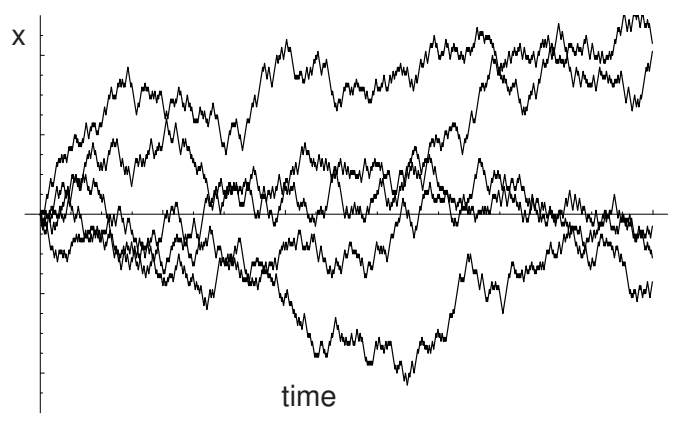

Fig. 4 Five different realizations of a random walk on a one-dimensional lattice illustrating the fractal nature of the trajectories, which in the continuum limit are continuous but non-differentiable.

\section{The art of mapping: from Brownian motion to quantum mechanics and back}

From what was just said, it is natural to expect that the Wiener process finds applications in many different areas far beyond the proper domain of Brownian motion, which is indeed the case [93]. Here we restrict ourselves to a few examples. Among physicists even better known than the Wiener measure Eq. (12) is the Feynman path integral [36], which expresses the propagator or Green function of a non-relativistic free particle in quantum mechanics as a path integral as in Eq. (12) but with an imaginary exponent. More precisely, the prefactor $-1 / 2 D$ is replaced by $i / \hbar$. Within the path integral picture one can interprete the uncertainty relation of quantum mechanics as the analog of the diverging apparent velocity of the Brownian particles that worried experimentalists before 1905. Note that the exponent in Eq. (12) has the form of the Lagrangian of a classical free particle. This analogy can be extended [94] to include a potential energy $-U(x)$ under the integral in the exponent of the Wiener measure. The corresponding Feynman path integral (the Feynman-Kac formula) can intuitively be understood as an analytic continuation of this extended Wiener measure in the complex plane, but a proper mathematical formulation is more subtle [93]. The diffusion (or Fokker-Planck) equation corresponding to the extended Wiener measure is Eq. (4) extended by a term $-U(x) n(x, t)$, corresponding to an annihilation probability. The diffusion equation corresponding to the Feynman-Kac propagator is of course the Schrödinger equation (in imaginary time).

Well known to physicists, this workhorse of quantum mechanics was the starting point for the development of various analogies. Building on the pioneering works, many important applications of path integrals have been developed in statistical physics and quantum and classical field theories, as well as in polymer physics and finance (where quantum mechanics was essentially mapped back onto Brownian motion), each 
field contributing its own characteristic difficulties to the basic formulation. Out of the innocent jittering of a pollen grain has grown a universal and powerful tool of theoretical physics, which has helped to unravel intimate connections between what seem to be vastly different phenomena at first sight.

We briefly sketch some key developments in the application to static conformations of flexible polymers. If segments of a flexible polymer could freely pass through each other, the Wiener process (in this context known as the Gaussian chain) would indeed provide an excellent coarse-grained description of the conformation if time $t$ is identified with arclength $s$ so that $6 D t$ is replaced by the mean-square end-toend distance $\left\langle R^{2}\right\rangle$ of the polymer. In particular, the Stokes-Einstein relation is translated into the "rubber" force-extension relation

$$
\frac{\partial\langle R\rangle_{F}}{\partial F}=\frac{\left\langle\boldsymbol{R}^{2}\right\rangle}{3 k_{B} T}
$$

which is easily recognized as the soft-matter analog of the Curie law for the inverse-temperature susceptibility of a paramagnet. Both become "stiffer" with increasing temperature, due to the disarranging action of thermal fluctuations. However, except under so-called $\theta$-conditions, where the self-avoidance of the chain is screened by an effective self-attraction, the simple random walk is unrealistic because of this idealization. Real polymers conformations cannot intersect and are characterized by self-avoiding random walks. The Edwards model $[95,96]$ represents self-avoidance by substituting the potential in the extended Wiener measure by a singular repulsion $U(\boldsymbol{r}) \propto \delta\left[\boldsymbol{r}(s)-\boldsymbol{r}\left(s^{\prime}\right)\right]$. This model has been the starting point of much theoretical work in polymer physics [97] and has been generalized to other self-avoiding manifolds [98]. It is amenable to a rigorous treatment with perturbation series and renormalization methods based on the path integral [97,99]. Notably, as has been pointed out by de Gennes [95], the problem of a self-avoiding random walk is related to critical phenomena, as it can also be mapped onto the $n \rightarrow 0$ limit of a $O(n)$-field theory. Figure 5 shows snapshots of fluorescently labeled DNA molecules adhering to a two-dimensional lipid membrane [100]. Measurements of the radius of gyration $R_{g}$ of the polymer have very well confirmed the prediction $R_{g} \propto L^{3 / 4}$ for the ("super-diffusive") conformation of a self-avoiding random walk in two dimensions. However, the interest of physicists for DNA is by no means limited to such very coarse and generic properties. Over the last 10 years DNA has become a favorite model system for a whole new field of investigation one might call "bio-polymer physics" [101-104].

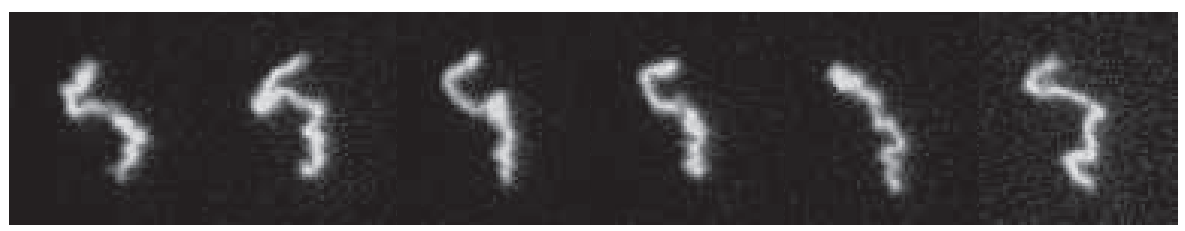

Fig. 5 Snapshots of fluorescently labeled DNA molecules adhering to a two-dimensional lipid membrane. Courtesy of J. Rädler and B. Maier [100].

Self-similar renormalized models are mathematically convenient but fail to indicate where the simplified universal representation breaks down. If one needs to incorporate more details, one has to resort to the level of the Chapman-Kolmogorov equation, Eq. (3), or master equation, Eq. (9). The latter has many features in common with the second quantized Schrödinger equation for many-particle quantum systems. In particular, both are linear differential equations of first order in time. Since each of the possible states $\alpha$ of the system can be labeled by a set of occupation numbers $n(x, t) \rightarrow\left\{n_{i}(t)\right\}$ (e. g. of lattice sites, energy states, chemical states, etc.), one is immediately led to a formulation in terms of an operator algebra in analogy with the second quantized formulation of quantum mechanics (for a short review and a list of references see e. g. [105]). Upon introducing boson ladder operators with the standard commutation relation $\left[a_{i}, a_{j}^{\dagger}\right]=\delta_{i j}$, one can define Fock states $|\alpha\rangle=\prod_{i}\left(a_{i}^{\dagger}\right)^{n_{i}}|0\rangle$ via acting with the ladder operators on 
the "vacuum" state $|0\rangle$. Then the master equation for the probability $P\left(\left\{n_{i}\right\}, t\right)$ of finding the system in the state with $\left\{n_{i}\right\}$ particles on site $\{i\}$, respectively, is equivalent to a Schrödinger equation in imaginary time

$$
\frac{d}{d t}|\Psi\rangle=-\mathcal{H}|\Psi\rangle
$$

for the state vector $|\Psi\rangle=\sum_{\alpha} P\left(\left\{n_{i}\right\}, t\right)|\alpha\rangle$. Of course, there are also differences from quantum mechanics. Most importantly, the "Hamiltonian" $\mathcal{H}$ is in general non-Hermitian. Still, most if not all many body techniques can be applied. In particluar, it is possible to transform the second quantized Hamiltonian into a field theory upon using coherent-state path integrals [65].

The similarities of the independently developed formalisms have led to a successful description of classical statistical systems far from equilibrium [105]. They not only allow for an application of field theoretical methods but also for a fruitful exchange of results and concepts between seemingly unrelated phenomena in quantum and classical physics. Very recently, it was e. g. suggested to represent gene expression as a many-body quantum problem [106], which leads to such unexpected analogies as that between the stochastic dynamics of a single gene switch and the quantum mechanics of the spin-boson model of a two-site polaron.

\section{A noisy world: the ubiquity of stochastic processes in biology}

\section{Genetic drift and the theory of neutral evolution}

Today we live in a "noisy world" where it is difficult to find a quiet place to contemplate. Noise in this context is simply unwanted sound and certainly deleterious. However, at the very origin of our lives are random processes, i. e. noise. Luckily, one should say, because otherwise we would largely be deprived of our individuality and doomed to live in a world of clones. This insight we owe to Mendel, who deduced from his experiments with pea flowers the existence of genes present as pairs of alternative forms (called alleles) in each (somatic) cell. He assumed that reproduction happens in a two step process, where equal separation of these gene pairs into the gametes (egg and sperm) is followed by random fertilization, i. e. random union of one gamete from each parent to form the first cell (zygote) of a new progeny's individual. This process is random precisely because there is Brownian motion on a molecular scale.

Starting at about the same time as Einstein's fundamental paper on Brownian motion the re-discovery of the Mendelian laws prompted the emergence of a new discipline, now known as population genetics (for an historic account see e. g. Ref. [107]). Pretty early on it was realized that random fertilization in finite populations leads to a phenomenon called "genetic drift" [108]. To illustrate this point let us look at the following toy model of population genetics (see Fig. 6), where one considers only one gene locus in a population of diploid ${ }^{12}$ organisms that has two alleles $A$ and $a$. We assume that each individual releases the same number of gametes, so that the allelic frequency in the population of gametes and the parental population are identical. From this large pool of gametes the next progeny is obtained by randomly chosing $N$ pairs of gametes ("random mating" or "panmixia"). In the language of sochastic processes this is a sequence of $2 N$ independent Bernoulli trials. If we denote by $x$ the relative frequency (probability) for the allele $A$ in the parental population, the probability distribution for the number $n$ of alleles $A$ in the next generation is given by the binomial distribution

$$
B(n, N)=\frac{N !}{n !(N-n) !} x^{n}(1-x)^{N-n} .
$$

Therefore, even if there are no deterministic evolutionary forces acting on a population (such as natural selection), sampling errors during the production of zygotes may cause a change in the frequency on a

12 The genome is made up of one or more extremely long molecules of DNA that are organized into chromosomes. The body cells of most plants and animals contain two genomes. These organisms are called diploid. For more details the reader is referred to a recent edition of some textbook in genetics, e. g. [109]. 
particular allele in a population; this phenomenon is called genetic drift. As can be read off from the variance of the binomial distribution, $D(x)=x(1-x) / 2 N$, genetic drift is expected to be large in small populations and vanishes for infinite population size. In the language of Brownian motion "genetic drift" is actually no drift at all, but a random walk of the stochastic variable $x$ (gene frequency). Two examples of such walks either leading to extinction or fixation are shown in Fig. 7.

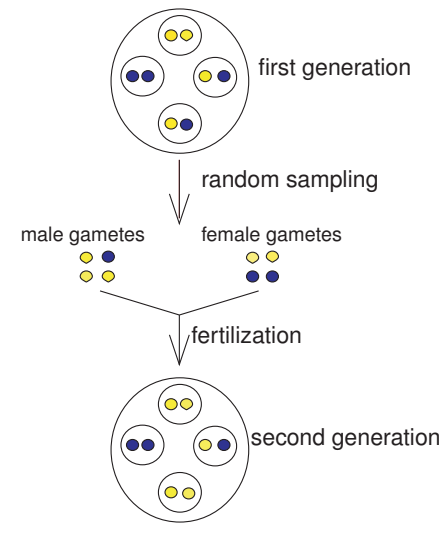

Fig. 6 Panmixia: a toy model of population genetics.

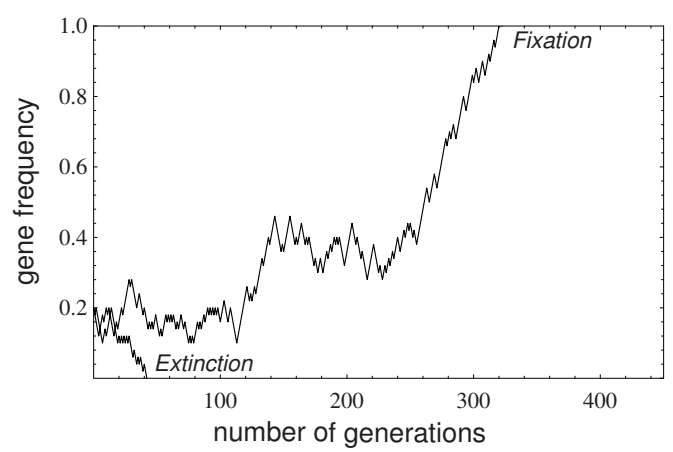

Fig. 7 Genetic drift. Some representative sample paths for random gene frequency drift.

In complete analogy to Einstein's formulation of Brownian motion one can now consider an ensemble of populations and derive a differential equation for the conditional probability density $n(x, p ; t)$ that the gene frequencs lies between $x$ and $x+d x$ at time $t$, given that the initial gene frequency was $p$. It is interesting to note that such a formulation was given by Fisher [108] without being aware of parallel developments in physics. The corresponding Fokker-Planck equation reads

$$
\frac{\partial n(x, p ; t)}{\partial t}=\frac{1}{2} \frac{\partial^{2}}{\partial x^{2}}[D(x) n(x, p ; t)] ; \quad \text { with } \quad D(x)=x(1-x) / 2 N,
$$

which can be interpreted as a diffusion process with progressively slower motion near the boundaries of gene frequency space, i. e. $x=0$ and $x=1$. Similar models with spatially varying diffusion coefficients have more recently been studied in the context of the voter model $[110,111]$ and of some catalysis reaction models at surfaces [112].

An essential feature of the random "drift" in gene frequency space is that there are boundaries. Once a given allele $A$ reaches $x=0$ it becomes extinct and the random process stops. Similarily if the allele $A$ reaches a frequency of $x=1$ it is said to be fixed (or equivalently allele $a$ becomes extinct). In other words, what we are facing here is a diffusion process with two absorbing boundaries. One may now ask the following questions. What is the time evolution for the distribution of unfixed classes with $0<x<1$ ? How likely is it for an allele to become fixed and what is the statistics of the number of generations until extinction or fixation of a certain allele occurs? Both of them can be answered by analyzing the FokkerPlanck equation Eq. 16, whose application to population genetics has been pioneered by Kimura, one of the founding fathers of the neutral theory of evolution ${ }^{13}$. He showed [113] that in the asympotic limit of a large number of generations the distribution function of the unfixed classes becomes flat and decays exponentially as $n(x, p ; t)=6 x(1-x) \exp (-t / 2 N)$. For answering the second question there is an alternative route using concepts from game theory. To see this let us shortly digress to a phenomenon called "gambler's ruin". Say the game simply consists of repeatedly throwing a fair coin. Your initial capital is $p$ (with $0<p<1$ ) and that of your opponent is $1-p$. The game is over when either one of

\footnotetext{
13 The neutral theory of evolution asserts that the great majority of evolutionary changes at the molecular level are caused not by Darwinian selecion but by random drift of selectively neutral mutations [107].
} 
you goes broke. What are your chances to win the game? The answer is simple (see e. g. Ref. [114]). Your chances are $p$, i. e. proportional to your initial capital, which can be rather low if you try to play against someone with large reserves ${ }^{14}$. This is exactly the game played by the genes in the above toy model of population genetics. Hence the probability that the allele $A$ eventually becomes fixed ("wins") is identical to its initial frequency $p$. But how long will it take to win? This is a first passage time problem (for a review see e. g. Ref. [115]) and can be solved by using a cousin of the Fokker-Planck equation, the backward Kolmogorov equation [116]. If one starts with just a single "invading" allele $A$ with a frequency of $p=1 / 2 N$ in a population of size $N$, one finds [117] that both the mean and the standard deviation of the time to fixation grow linearly with the size of the population.

This simple toy model of population genetics with one gene locus, two alleles and random mating is, of course, much to naive to account for the variety and complexity of life forms on our planet. On a molecular level, there are complex changes in DNA sequence, which may either be random-like point mutations and frameshifts or more systematic cut-and-paste mechanisms like insertion sequences and transposons [118]. On the phenotype level, there are interactions among the organisms in a population and with the surrounding environment, which may itself be dynamic and noisy. The fitness, i. e. the number of expected offsprings, of an individual is a (highly non-linear) function of such interactions. The way from genotype to a phenotype is paved by networks of coupled biochemical reactions and feedback signals. The list goes on and would lead us considerable off track; for more information the interested reader is recommended to consult some recent introductory review articles $[119,120]$.

\section{Stochasticity in chemical reactions: the reign of small numbers}

The machinery of biological cells consists of networks of molecules interacting with each other in a complex manner. Many of these interactions can be described as chemical reactions, where the intricate processes which occur during the encounter of two molecules are reduced to reaction rates ${ }^{15}$. For example, an autocatalytic reaction for an enzyme $E$ is modeled as a probabilistic self-replication process $E \rightarrow 2 E$, which occurs at a constant probability per unit time (reaction rate) $k$. For high concentrations of the enzyme, a standard approach in chemistry is to write down rate equations for the time evolution of the average concentration $\bar{n}(t)$

$$
\frac{d \bar{n}(t)}{d t}=k \bar{n}(t) .
$$

Such an approach evidently assumes that the time evolution of a chemically reacting system is both deterministic and continuous. However, the actual process is neither of both since molecular population levels change stochastically and by discrete integer amounts.

Obviously, a deterministic description is applicable only if deviations from the average number of molecules are negligibly small. As noted long ago by Delbrück [123] the validity of this assumption breaks down in small chemical systems, where the concentrations of reacting species are extremly low and the statistical fluctuations of atomism can not be avoided. Then, instead of the average concentration $\bar{n}$ one now has to consider the full probability distribution function $P_{n}(t)$ of the number of enzymes $n$ at a given time $t$. This function plays the same role as the particle density $n(x, t)$ in Einstein's treatment of Brownian motion or the probability density of gene frequency $n(x, p, t)$ in our discussion of genetic drift. Instead of continuous random variables (particle position, gene frequency) we are now dealing with the discrete random variable $n(t)$, the number of enzymes at time $t$. The time evolution for $P_{n}(t)$ can immediately be written down if one pictures an ensemble of enzymatic reactions, where $P_{n}$ is the relative frequency of samples in the ensemble with exactly $n$ molecules. The frequency $P_{n}$ (population size)

\footnotetext{
14 But history teaches us that even the big players with large reserves may sometimes stumble and fall [7].

15 Reaction rate theory was pioneering by Kramers [121]; for a recent review see e. g. [122]. He considered a simplified model system where a Brownian particle is trapped in a one-dimensional well representing the reactant state which is separated by a barrier of finite height from a deeper well signifying the product state.
} 
of this particular class is reduced by each self-replication process within the class and enhanced by each self-replication processes in the neighboring class. Then the time evolution of $P_{n}(t)$ can be written as a "gain-loss" equation

$$
\frac{d}{d t} P_{n}(t)=(n-1) k P_{n-1}(t)-n k P_{n}(t)
$$

a specific example of the master equation introduced in section 4 .

How are the results of a stochastic description, Eq. 18, different from a deterministic approach, Eq. 17? The most striking one is, of course, that despite the exponential growth, $n(t)=n_{0} \exp (k t)$, promised by a deterministic description there is a chance to loose it all, $n(T)=0$ (gambler's ruin). Other features of the stochasticity become evident upon solving the master equation for reactions initiated by one particle. Then the probability distribution for large times $t$ has the asymptotic form

$$
P_{n}^{1}(t)=\frac{1}{\bar{n}} e^{-n / \bar{n}} \quad \text { with } \bar{n}=e^{k t},
$$

which is by no means normal, but exponential. Note that at all times the probability of finding a single particle is greater than the probability of finding any other specified number of particles. In addition, the standard deviation is idential to the mean of the distribution. Delbrück also points out that there are strong fluctuations in the waiting time for attaining a certain threshold amount of reaction products [123].

An even more dramatic illustration for the importance of stochasticity in chemical reactions is given by the pair annihilation process $A+A \rightarrow 0$ in one-dimensional systems. The rate equation, $d n / d t=-k n^{2}$ would predict an algebraic decay as $n(t) \sim t^{-1}$. However, the actual asymptotic decay is much slower, $n(t) \sim t^{-1 / 2}$. This slow decay can be traced back to the reentrance property of random walks in less than two dimensions, which implies that within a volume of order $\sqrt{D t}$ all particles are annihilated with probability one [124]. A more sophisticeted line of argument uses methods adapted from quantum field theory [125]. The pair annihilation process is the simplest member of a broad class of models known as diffusion-reaction models exhibiting absorbing states, recently reviewed in Ref. [126]. That such systems show unusual dynamics becomes obvious by asking the innocent looking question: How does a system evolve towards its steady state? Sometimes the answer is quite simple and the relaxation process is merely an exponential decay. If the deviations from equilibrium are small Onsager's regression hypothesis [127] asserts that the relaxation is governed by the same laws as the fluctuations in equilibrium. But this hypothesis certainly fails for systems with an absorbing steady state. Here there are no fluctuations in the steady state but the approach towards the absorbing state is critical in the sense that it exhibits slow power-law decay and universal scaling behavior [128].

Before getting too much sidetracked let us come back to biology. Here it has been emphasized recently that living cells possess very low copy numbers of many components, including DNA and important regulatory molecules [129]. As a consequence, stochastic effects such as those illustrated above may play a key role in biological processes. A quite striking example are observations $[130,131]$ that clonal populations of cells (i. e. genetically identical cells) exhibit substantial phenotypic variation. Recent experiments even allowed to distinguish between the stochastic noise inherent in the chemical reactions controlling gene regulation and fluctuations in the number of other cellular components [132]. These observations are only the tip of an iceberg. "Noisy" mechanisms may well be rather the rule than the exception in the molecular world of a cell [133].

\section{The Brownian particle: probe and effigy of the intangible}

\section{Probing molecular fluctuations with Brownian particles: "the buoy in the surge"}

Precisely in the way proposed by Einstein [2], Brownian particles have ever since been used as probes for the molecular motion of the surrounding medium: recently in particular for probing the local viscoelastic 
response of soft, often biological, materials, where elastic moduli are of the order of $k_{B} T$ or even due to $k_{B} T$ (i. e. of entropic origin). A zoo of methods based on Brownian motion of tracer particles has been invented: (i) particle-tracking techniques ${ }^{16}$ that record the path of one particle $[17,135,136]$ or the correlated fluctuations of two particles [137, 138]; (ii) diffusing light spectroscopy [139, 140], which iterates our basic theme by exploiting not only the diffusion of colloids but also the diffusion of light they scatter; (iii) Fourier-imaging-[141] and fluorescence-[142] correlation-spectroscopy, which are more similar to classical dynamic scattering techniques; etc. According to the fundamental connection between fluctuations and response provided by Eqs. (2), (5),(6) the fluctuations measured by these passive techniques contain the same information as obtained by active microrheological linear-response measurements (e. g. [143]). One can roughly classify the passive methods into those working in Fourier space ("scattering") and those working in real space ("microscopy").

The basic principle of scattering methods probing Brownian dynamics is well illustrated by the example of particle sizing, which is a widely applied standard method. One shines a laser beam of wavelength $\lambda$ at a dilute suspension of $N$ identical particles in a viscous medium and records the light intensity scattered at an angle $\theta$ to the incident beam. The auto-correlations of the scattered light then decay proportional to (the square of) the dynamic structure factor

$$
S_{q}(t)=\frac{1}{N} \sum_{i j}\left\langle e^{i \boldsymbol{q} \cdot\left[\boldsymbol{r}_{i}(t)-\boldsymbol{r}_{j}(0)\right]}\right\rangle \stackrel{\text { dilute }}{\sim} \frac{1}{N} \sum_{i}\left\langle e^{i \boldsymbol{q} \cdot \delta \boldsymbol{r}_{i}(t)}\right\rangle=e^{-q^{2}\left\langle\delta \boldsymbol{r}_{i}(t)^{2}\right\rangle / 6}=e^{-q^{2} D t} .
$$

Here, $\boldsymbol{q}$ is the scattering vector, $q \equiv|\boldsymbol{q}| \equiv 4 \pi \mathrm{n} \sin (\theta / 2) / \lambda$ with $\mathrm{n}$ the refractive index, and we used that dilute particles are uncorrelated. Applying Einstein's Eq. (5) together with the Stokes relation $\zeta=6 \pi \eta a$ for the friction of a spherical particles in a viscous solvent, one can determine the diameter $a$ of the particle or alternatively the vicosity $\eta$ of the solvent.

\section{Brownian particles in complex fluids: "the buoy in the pudding"}

Although the idea of exploiting such kind of methodology to measure material properties in small samples and even inside living cells is also a rather old one [144], its applications mulitplied over the last two decades or so with surgeing interest in highly disordered, soft, and/or biological matter. Reports of substantial deviations from Eq. (5) in such systems abound. A common observation is that of superdiffusive $(\alpha>1)$ or subdiffusive $(\alpha<1)$ dynamics, which was studied theoretically in great detail [72], and which is characterized by

$$
\left\langle\delta \boldsymbol{r}^{2}\right\rangle \propto t^{\alpha} \quad \text { with } \alpha \neq 1 .
$$

Such "strange kinetics" [145] has even prompted the development of a dedicated "fractional calculus" [146-148]. It appears in many areas of science (nonlinear dynamics, growth phenomena, fluctuating manifolds, etc.). A simple way to generate such anomalous diffusion is by assuming jump rates $\varphi_{\tau}(\xi)$ in Eqs. (3) (10) that decay too slowly with $\xi$ (i. e. like $\xi^{-\mu}, 1<\mu<3$ ) for the ordinary central limit theorem to apply, or, equivalently, for constant step length by a broad waiting time distribution. For Brownian particles, it naturally arises as intermediate asymptotics whenever the solvent is not a simple liquid but a complex (viscoelastic) fluid with some internal structure that exhibits long-range spatial or temporal correlations; i. e. it has more to do with a complicated material property of the solvent than with Brownian motion as such, and thus leads us slightly off-topic. Biological cells certainly provide us with ultimate complexity, which makes deviations from Eqs. (5), (20) anything but surprising. It is therefore very suggestive to interprete subdiffusion in cells as a consequence of cytoplasmic heterogeneities and crowding $[149,150]$, but attempts to explore the actual connection are still limited to model systems $[151,152]$. If

\footnotetext{
16 Roughly speaking, even the latest computer-aided confocal video-microscopy setup for particle tracking is but a costly late descendant of the technique pioneered by Henri in 1908 [134], when he first put a film camera onto a microscope to record the wild paths of Brownian particles in order to scrutinize Einstein's prediction, Eq. (5).
} 
not due to complex (biological) organization, one generally expects long-range correlations to be selfsimilar, arising e. g. as a consequence of a closeby equilibrium critical point, turbulence, or some other non-equilibrium structure. A nice example of temporal correlations is provided by simple shear flow: it gives rise to a peculiar cooperation of statistical mechanics and fluid mechanics known as Taylor diffusion [29, 153], which effectively speeds up the diffusion of (dilute) suspended Brownian particles. Stirring your coffee thus helps twice to distribute the suger, via turbulent convection on large scales and via the induced superdiffusion on shorter scales. A common paradigm based on spatial correlations is diffusion in a porous medium with a very broad pore size distribution, idealized as diffusion on a fractal $[154,155]$. More intriguing from a theoretical perspective is the case, where subdiffusion arises without any nontrivial structures in configuration space as a consequence of a complicated phase-space structure. This kind of "dynamically self-generated" subdiffusion is often characterized as glassy dynamics (see section 3 and below).

\section{Long-time tails: "the buoy's bow wave"}

Maybe most suprisingly, already the dynamics of a single Brownian particle in a simple Newtonian solvent has some subtle properties not anticipated by Einstein and Perrin. In his derivation, sketched above, Einstein implicitely assumed $\varphi_{\tau}(\xi)$ to have properties such that the expansion of the Chapman-Kolmogorov equation, Eq. (3), nowadays known as Kramers-Moyal expansion, can be truncated after the $\nabla^{2}-$ term, thus yielding a simple hydrodynamic equation, the diffusion equation, Eq. (4). Such conditions are mathematically realizable $[27,28,156]$ but not necessarily physically relevant $[116,157]$. They amount to a description of Brownian motion as a Gaussian Markov process (a notion that did not yet exist in 1905). Although this may seem to be a very plausible assumption given the law of large numbers and the strong scale separation between the Brownian particle and the molecular scale, it only holds to a first approximation, or for $t \rightarrow \infty$. Hydrodynamic conservation laws, as e. g. contained in the Stokes equation for the solvent, induce long-range auto-correlations that effectively retard the approach to the Markov limit. At any finite time, they contribute transient corrections, so-called long-time tails, to Eq. (4) and moreover render Eq. (3) and the subsequent expansion ill defined [158-160] (for overviews see e. g. [157, 161] and references therein). A quick derivation exploits Eq. (6). Recalling that the dynamics of the solvent is itself governed by a diffusion equation (see footnote 2 , section 2 , above) we conclude that the momentum of the Brownian particle is diffusively dissipated, hence shared with a growing solvent volume $\Delta(t)^{3} \propto(\nu t)^{3 / 2}$, so that the velocity auto-corelations in Eq. (6) decay like $t^{-3 / 2}$. The effective diffusion coefficient $\left\langle\delta \boldsymbol{r}^{2}(t)\right\rangle / 6 t$ at finite time $t$ is obtained by setting the upper limit of integration in Eq. (6) to $t$, which immediately gives $[157,162]$

$$
\left\langle\delta \boldsymbol{r}^{2}(t)\right\rangle=6 D t[1-\sqrt{\theta / t}+\ldots],
$$

where $\theta$ is a hydrodynamic time scale that depends on $\zeta$ and the particle/solvent mass ratio. Thus, normal Brownian motion actually is slightly faster than diffusive, Einstein's formula is only slowly approached, and there is no simple general way to ammend the diffusion equation to take this into account. The somewhat uncomfortable picture [157], which first emerged from calculations for dense gases based on the Boltzmann equation [158], has sometimes been summarized as Dorfman's lemma [163]: all relevant fluxes are nonanalytic functions of all relevant variables. Neither does a gradient expansion of the diffusion current exist (the Burnett-coefficients appearing in the expansionn are found to be infinite), nor do density expansions exist of the diffusion coefficient and other transport coefficients as one might expect in analogy with the virial expansion of thermodynamics. Luckily, the corrections in Eq. (22), though of fundamental interest, are in general numerically too small to spoil seriously any of the numerous applications mentioned above. We note in passing that related problems arise in statistical mechanics derivations à la Einstein and Smoluchowski of hydrodynamic boundary conditions (e. g. "no-influx" for a tagged Brownian particle in a colloidal suspension [164] or "no-slip" in a Newtonian fluid [165-167]). Again, typical microrheological applications may well get by without such subtleness [168]. However, one should be aware that tails 
do seriously affect Brownian motion in lower dimensions, leading to "hydrodynamic suicide" [51] in two dimensions. Even without considering solvent hydrodynamics, due to the otherwise subdominant longrange concentration patterns ("colloidal tails") implied by Eq. (4), Brownian motion in 1-dimensional pores exhibits anomalous, subdiffusive dynamics, so-called single-file diffusion [169].

\section{Interacting Brownian particles: the upscaled microcosmos}

Apart from providing us with a large assortment of colloidal particles to probe the molecular world, the methods pioneered by Perrin, Svedberg, and others moreover opened the way to designing an effigy of the molecular world on the colloidal scale [170-172]. The natural laboratory of Gouy was successfully transformed into an artificial microcosmos [25], which obviously leads us slightly off-topic, again. Svedberg's ultracentrifuge helped to solve one of the big experimental challenges in colloid preparation: to prepare samples that have sufficiently well-defined properties, in particular a monodisperse size distribution to mimic as closely as possible the sameness of atoms. This is hard work: starting with one kilogram of gamboge, which is a gum resin named after Cambodia (from where it was originally imported), Perrin "obtained after several months of daily operations a fraction containing several decigrams of grains with a diameter of approximately three-quarters of a thousandth of a millimetre" [5]. But monodisperse micronsized particles were a crucial prerequisite for the progress of colloid science. With an enormous toolbox at hand, chemists are now able to design almost any colloidal interactions one may wish for, far beyond what can be found among naturally occuring molecules.

This gave experimentalists a formidable playground for testing highly idealized theoretical pet-models for many-body problems, such as the hard-sphere system and its various extensions. As anticipated by van der Waals in the 19th century, much of the packing structure of liquids can be understood from the hard-shpere like mutual repulsions between their constituent molecules [173]. Hard spheres also provide a beautifully simple example (paradigmatic for more complicated soft condensed matter systems [15] including living cells [174]) how macroscopic order emerges from microscopic chaos, namely the stochastic Brownian motion of the individual spheres [175]. Numerical simulations [176, 177] and experiments [178] have established that above a sharply defined volume fraction $\phi=0.494$, Brownian fluctuations drive the particles into a crystal, because they gain space to wobble around when they arrange themselves on a (virtual) lattice ${ }^{17}$. Microgravity experiments [179] suggest that this crystal is made out of randomly stacked hexagonally close-packed planes. It becomes space filling at $\phi=0.545$ and seems to develop a slight preference for face-centered cubic organization if it is compressed towards closest packing [180] at $\phi=0.74$. Colloidal crystallization has gained additional interest from those trying to crystallize proteins for structure analysis [181]. While, in this context, colloids are currently still mostly playing their usual role as visible model systems for globular proteins [182], programmable protein expression might in the future well overcome chemistry as the more powerful machinery for providing colloid physics with the ultimate design precision.

If quickly quenched to volume fractions $\phi>0.58$, hard spheres will provide yet another interesting and suprising feature closely related to our central theme: by their Brownian motion they trap themselves in an arrested non-equilibrium amorphous state that looks like a liquid but feels like a solid, and which may substantially delay or even impede crystallization: the colloidal glass ${ }^{18}$. From mode-coupling theory [185] a very characteristic (self-generated) subdiffusive behavior [186] during the critical slowing-down of the Brownian motion near this transition was predicted. This was beautifully confirmed (see e. g. Refs. [187, 188]) for collective density fluctuations by light scattering [189], and for tagged particles by numerical simulations [190]. At least for hard-sphere like systems the theory can be regarded as legitimate — though,

\footnotetext{
17 This illustrates the paradox that global order may help to increase individual freedom.

18 To be fair to the photon [183], we note aside that not only diffusing Brownian particles may come to a halt, but also diffusing light. Recently, colloidal crystallization has gained some attention as a promising method to produce photonic crytals [184], which may become an attractive technology in the future (currently all the rage: hiding light under a bushel).
} 
unfortunately, not completely rigorous ${ }^{19}-$ sucessor of Eq. (4) towards a more microscopic picture. It starts from a microscopic expression for the normalized dynamic structure factor $\phi_{q}(t) \equiv S_{q}(t) / S_{q}(0)$, cf. Eq. (20), in terms of generalized fluctuating particle currents $J$

$$
\frac{\partial \phi_{q}(t)}{\partial t}=-D(q) q^{2} \phi_{q}(t)+\int_{0}^{t} d \tau\left\langle J_{q}(t-\tau) J_{q}(0)\right\rangle \phi_{q}(\tau) .
$$

For short times and $q \rightarrow 0$, this reduces to the Fourier transform of the diffusion equation. The kinetic coefficient $D(q)$ generalizes the gradient diffusion coefficient $D=D(q \rightarrow 0)$ to finite wave vectors. For long times, it becomes effectively reduced due to the term reminiscent of Eq. (6), through which the system remembers its past evolution (e. g. its long-time tails). To make practical use of Eq. (23), the microscopic memory kernel containing the Brownian fluctuations $J$ has to be expressed in terms of the hydrodynamic variables $\phi$, which involves the uncontrolled mode-coupling approximation [187,192,193]. Yet, after iterative solution, Eq. (23) predicts in impressive detail how, upon increasing either the attraction or the volume fraction, the simple Brownian dynamics of a fluid suspension described by Eq. (4) gives way to slow glassy dynamics, and eventually to two disticint non-ergodicity transitions into two fundamentally different glassy states [194, 195].

\section{Fluctuating manifolds: Brownian particles with soft internal modes}

Just as it is commonplace that history repeats itself, re-inventing the wheel is an everyday experience in science. So it could have been expected that researchers studying red blood cells under the miscrosope would be tempted to ascribe their flickering shape undulations to animate causes, thereby repeating the initial misinterpretation of Brownian motion as a sign of life by (most of) its early discoverers (see Ref. [196] for a discussion of the history). The story of Brownian motion was about to repeat itself for Brownian particles with soft internal degrees of freedom. Today, cell flickering is understood to be a physical phenomenon [196] that has an important biological implication, tough: the reduction of unfavorable adhesion via the so-called Helfrich repulsion [197] caused by the thermal undulations of the cell membrane. Similarly, the thermal conformational wiggling of the polymeric constituents of the cytoskeleton has been pinpointed as the origin of some characeristic viscoelastic behavior $[168,198,199]$.

\section{Brownian undulations ...}

For fluctuating manifolds, not only the center of mass position or overall orientation but an (ideally) infinite number of internal modes are excited into a persistent dance by thermal forces. Brownian fluctuations tend to "crumple" low dimensional manifolds [98]. Their free and driven ${ }^{20}$ conformational dynamics is the subject of a field of investigation that one might summarize by the term stochastic elastohydrodynamics. It is largely motivated by the current interest in soft and biological materials. Examples of fluctuating manifolds are flexible linear and branched polymers, microemulsions, foams, liquid crystals and numerous other soft surfaces, interfaces and membranes [96,98, 204-208], but also vortex matter, line liquids, magnetic domain walls and the like [209,210].

For the most natural case of an elastically uniform manifold of $n=1$ or $n=2$ dimensions [211], the force needed to excite internal modes will grow as a power law with the mode number $q$, equilibrium amplitudes $a_{q}$ and relaxation times will decrease as $\left\langle a_{q}^{2}\right\rangle \propto q^{-\beta}$ and $\tau_{q} \propto q^{-\gamma}$, respectively. For $\gamma>\beta-n$,

\footnotetext{
19 The worst theory apart from all others that have been tried from time to time [191].

20 Opening the Pandora box of non-equilibrium one enters the vast topic of pinned or driven fluctuating manifolds [200-203] that comprises a wealth of complex non-equilibrium phenomena in many areas of condensed matter physics, which we will not pursue any further, here.
} 
this is quickly translated into a subdiffusive ${ }^{21}$ mean-square displacement

$$
\left\langle\delta \boldsymbol{r}^{2}(t)\right\rangle=2 \sum_{q}\left\langle\boldsymbol{a}_{q}^{2}\right\rangle\left(1-e^{t / \tau_{q}}\right) \propto t^{(\beta-n) / \gamma}
$$

by transforming the sum into an integral. This is the appropriate generalization of Eq. (5) to real-space measurements of a small labelled patch on the manifold, which have indeed been very successfully applied to various biomolecules [212-214]. Similarly as for simple Brownian particles, Eq. (24) determines the decay of the dynamic structure factor for long times $(t \rightarrow \infty)$, when only the self-correlations of patches of dimension $q^{-1}$ matter, via Eq. (20). In contrast, for times shorter than the relaxation time of modes of wavelength $q^{-1}$, the fluctuating interference of light scattered from neighboring elements of the manifold within such patches has not yet averaged out and dominates the dynamic decorrelation of the scattered intensity. While the internal modes thus affect the decay law for long times, $S_{q}(t \rightarrow \infty) \propto e^{-\mathcal{D} q^{2} t^{\alpha}}$, they merely renormalize the effective diffusion coefficient for short times, $S_{q}(t \rightarrow 0) \propto e^{-\mathcal{D}_{q} t}$. Both limits encode information about the material properties of the manifold and the solvent in different combinations. (Which regime dominates the dynamic structure factor is determined by the elasticity of the manifold. For flexible manifolds the simple exponential behavior dominates, and for stiff ones the stretched exponential tail.) Under favorable conditions this can be exploited to probe these parameters [215] in analogy to the simple example of particle-sizing discussed above. Fluctuating manifolds thus add a new facet to the story of Brownian motion: subdiffusive behavior may not only arise due to complicated surroundings but equally well from (quite simple!) internal elastic degrees of freedom of the tracer. In particular in presence of external forces this opens up the possibility of rich non-equilibium Brownian dynamics even for such seemingly trivial objects as a thread in a Newtonian solvent [216]. If the long-range hydrodynamic self-interactions, mentioned above in connection with the long time tails, need to be included, they lead to considerable complications, as e. g. for flexible polymers [96] and for membranes [217], even in equilibrium.

\section{... in complex fluids}

Increasing the complexity by one more step, one can combine both extensions of simple Brownian motion, internal elastic modes and a complex solvent. Consider a long (tagged) polymer embedded into a dense melt or solution of other (identical) polymers. The question how the polymer diffuses through the medium is closely (but by no means trivially) related to the question how the whole medium is able to flow. Both are addressed by reptation theory [96, 204,218], the prevailing phenomenological idea how this involved many-body problem can be disentangled and reduced to a description based on a single polymer laterally confined to an effective tube. The concept is popular for its intuitive elegance and its ability to rationalize data for the elastic modulus and the viscosity. One imagines the polymer as a random walk caught in a cage shaped like a curly tube of mean-square end-to-end distance $\left\langle R^{2}\right\rangle$ proportional to its length $L$. Taking into account that it has to diffuse back and forth all the way along the tube to completely disengage from it, and that the diffusion coefficient $D_{\|}$for diffusion along its own contour is inversely proportional to $L$, one deduces the disengagement time

$$
\tau_{r} \propto L^{2} / D_{\|} \propto L^{3}
$$

For shorter times, due to multiple inter-polymer collisions [99], semidilute solutions or melts respond elastically to shear, like a rubber with an elastic modulus of $k_{B} T$ per statistically independent segment of the tube. These segments (of length $\left\langle R^{2}\right\rangle / L$ ) act as its effective unit elements. At times longer than $\tau_{r}$, entanglements can relax, and the whole medium can flow like a liquid. The large time $\tau_{r}$ therefore also fixes the ratio of the medium viscosity at long times to the shear modulus at shorter times, which implies that polymer melts and solutions are soft but tenacious, or "gloop". Support for the tube, intuitive starting point

21 If this inequality is violated, one does not obtain superdiffusion but the dynamics is dominated by the lowest mode. 
of the reptation model, comes from direct visual observation of the Brownian motion of large biopolymers $[219,220]$. Towards a deeper understanding of how viscoelasticity emerges from the underlying basic laws of Brownian motion, microscopic approaches (theory [221] and simulations [222,223]) are currently pursued.
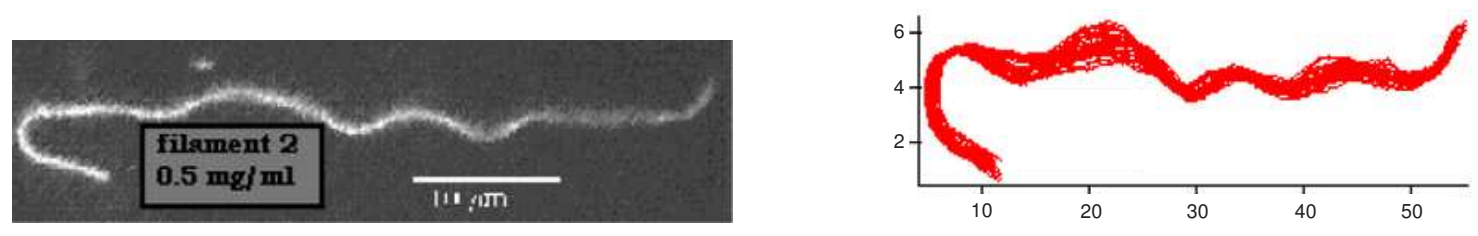

Fig. 8 Direct visualization of the tubes around actin filaments in F-actin solutions. Courtesy of J. Käs [219].

Over the last decades, membranes and polymers have become the major companions of the simple Brownian particle as paradigms of soft condensed matter, which is what we ourselves and many of our natural and technological aides of everyday life (foods, cosmetics, detergents, plastics ...) are made of. Rather than continuing this list ad infinitum, we close this section by pointing out an ironic twist in the recent history of Brownian motion: with the physics of fluctuating polymers and membranes reasonably well understood, physicists have at last turned their attention back to the possible animate sources of Brownian fluctuations. The idea is to study the additional effect of "active noise", such as the undulations induced by molecular pumps, molecular motors, and switching channels embedded into a membrane [224,225], a theme that can be iterated in many directions; e. g. towards so-called active gels [226-230], solutions of biopolymers mixed with molecular motors, in which rich structure formation (asters and spirals, etc.) has been observed in vivo and in vitro. Interestingly, the "noise" produced by the biological activity was predicted to lead to a thousandfold increase of the amplitudes of the long-time tails [231].

\section{Rectified Brownian motion: from "life force" to "living force" and back}

It is an amusing semantic detail from the history of science that kinetic energy was originally termed living force ("lebendige Kraft") by Helmholtz and his contemporaries, adopting Aristoteles' "vis viva". When Brown first observed the irregular motion of pollen granules, he was convinced that he had found animate objects driven by some kind of "life force" outside the realm of physics and chemistry, a vitalistic view still common at that time. He derived from Leeuwenhoek's terminology of "animalcules" (small animals) the name "molecules" for the rambling particles under his microscope. After their animal aura had been stripped off by the subsequent investigations, the term could be reused for those particles that carried the thermal living force then recognized as the mundane physical explanation of the hitherto mysterious life force.

This section will deal with the origin of motion in living systems and will bring us back once again to the "living force" as the "life force" responsible for directed motion on a cellular scale. The origin of motion of living organisms was already debated in the 3rd century B.C., when the anatomist and physician Erasistratos of Ceos associated muscle motion with the "spiritus animalis". He imagined it as some kind of fluid or gas, which he termed "pneuma", that flows through hollow nerves as pipelines and makes the muscles swell and shorten. This pneumatic idea survived in the scientific community for a very long time. It was only with the invention of the microscope by van Leeuwenhoek (1632-1723) that Swammerdam (16371680) was able to show that muscles contract at constant volume which invalidated pneumatic theories. Helmholtz in his famous paper about the law of energy conservation [232] may have been the first to 
emphasize that the mechanical energy produced by living organisms is just transduced chemical energy 22 . These ideas paved the way for a physical understanding of muscle contraction. A discussion of the early work on theoretical models, which already contains some of the ideas of more recent work discussed below, can be found in reviews of A.F. Huxley [233] and Hill [234].

Modern experimental techniques [235] have lifted the veils and allowed us to look at the causes of biological motion on a molecular scale. This resulted in the following picture [236]. Biological motion is caused by (nanometer sized) motor proteins, a highly specialized class of enzymes which are able to transduce the energy excess in the chemical hydrolysis reaction of ATP (adenosine-triphosphate) into mechanical work. An important subclass are cytoskeletal motors, which by sequence similarity are classified into three families (myosins, kinesins and dyneins). These motors "walk" along one-dimensional molecular tracks consisting of quite stiff protein fibres (F-actin and microtubules). They are involved in many biological processes essential for living organisms, such as mitosis and meiosis, as well as muscle contraction and intracellular transport.

\section{How individual motors work}

Looking at these systems with the eye of a theoretician, molecular motors reduce to microscopic objects (and as such they are subject to Brownian motion) moving uni-directionally along one-dimensional periodic substrates. The obvious question is: "What are the mechanisms which rectify Brownian motion and can explain such a uni-directional motion?" The answer is that there are actually a variety of mechanisms, different aspects of which are discussed in a series of recent reviews [237-241] (see also the article by P. Hänggi et al. in this volume [242]).

The essential idea can be summarized with the following rather elementary but illustrative example, known as the "flashing" ratchet [243-245]; see Fig. 9. Consider a Brownian particle subject to viscous damping which is moving along a track with a periodic, asymmetric potential $V(x)$ (with a sawtooth-like shape). Despite the spatial asymmetry of the potential, no preferential direction of motion is possible if in an isothermal environment only equilibrium fluctuations act on the particle. This argument goes back to Smoluchowski [246] (see also the Feynman lectures [1]) who showed that otherwise one would be able to construct a perpetuum mobile and violate the second law of thermodynamics.

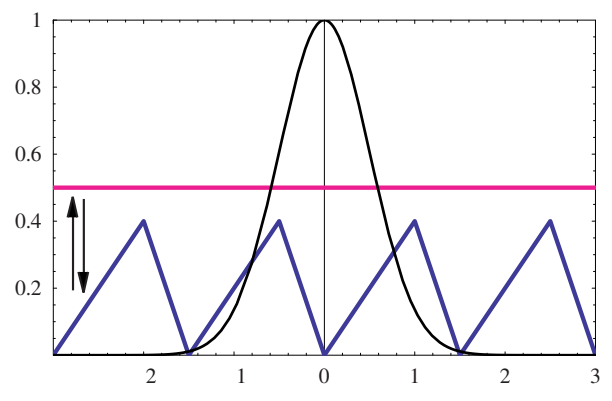

Fig. 9 Illustration of a "flashing ratchet". Upon switching a sawtooth-like potential on and off, a Brownian particle will move uni-directionally along a one-dimensional molecular track.

Thus, for generating uni-directional motion we obviously need an additional ingredient which drives the system out of equilibrium. In a "flashing" ratchet this is achieved by cyclically turning the potential on and off. The principle is easily explained. Say that the potential barriers are rather high compared to any thermal energy scale. Then, with the potential switched on, the Brownian particle is well localized in

22 "Es bleiben uns von den bekannten Naturprozessen noch die organischen Wesen übrig. ... [Tiere] verbrauchen also eine gewisse Quantität chemischer Spannungskräfte, und erzeugen dafür Wärme und mechanische Kräfte." At that time the term "Spannungskraft" and "lebendige Kraft" stood for what we nowadays call potential and kinetic energy, respectively. 
one of the potential minima. Upon turning the potential off, the Brownian particle starts performing a free one-dimensional random walk, such that after some time $\tau_{\text {off }}$ the probability distribution $P(x)$ for finding it at position $x$ is given by a Gaussian

$$
P(x)=\frac{1}{\sqrt{4 \pi D \tau_{\text {off }}}} \exp \left[-\frac{x^{2}}{4 D \tau_{\text {off }}}\right],
$$

where $D$ is the diffusion coefficient. When the potential is turned on again, the asymmetry of the potential acts as a rectifyer on the probability distribution function and causes net transport in one direction; see Fig. 9. In this sense Brownian motion acts as a "life force" ("Lebenskraft") for molecular scale engines. Note, however, that the energy to drive the transport does not come from the thermal noise but is provided when the potential is switched on and off.

Recently there have been several experimental ramifications of the "flashing ratchet" using colloidal particles [247,248]. With the advances in nanosciences and microfluidics there are by now many cleverly designed systems, classical as well as quantum, which utilize Brownian motion for generating transport, sorting particles and performing other tasks [249].

The "flashing ratchet" is, of course, not a realistic model for any kind of actual biological motor. It oversimplifies a variety of features of real biological engines. First, molecular motors are not structureless particles but proteins with a quite complex architecture and hence a large number of internal degrees of freedom. For example, kinesin is a dimer consisting of two globular head domains joined together by alphahelical coiled-coil. Second, the interactions of the motor protein with its fuel ATP and the molecular track are complex molecular processes, a full analysis of which would require an atomistic description including all the different types of forces between the chemical agents in a watery environment. To go beyond generic models making the proof of principle one has to design more elaborate models which account for the most important structural and chemical elements. What "most important" means varies from system to system. It depends on specific features of the particular engine, e. g. the type of coupling between the biochemical cycle and the conformational states of the protein ${ }^{23}$. Depending on the level of coarse-graining we may describe the forces acting on the degrees of freedom as systematic or stochastic. Systematic forces include viscous drag and "mechanical" forces acting on structural elements of the protein which are sometimes visualized as parts of macroscopic engines (springs, levers, joints, etc.). Stochastic forces may be classified as Brownian conformational fluctuations and stochastic chemical reactions. Both, of course, are just variations of the same theme. Their common cause is the chaotic dance at a molecular scale. The "flashing ratchet" and the "Possion stepper" emphasize these two sides of the medal. In the flashing ratchet all stochasticity results from Brownian conformational fluctuations in a potential landscape. In contrast, for the "Poisson stepper" (a textbook example for a discrete stochastic process), which advances step by step at constant rate, stochasticity results from the distribution of waiting times between the steps [250].

\section{Traffic jams in the cell}

There are several biological processes where a concerted action of molecular motors is of importance. A particulary prominent example is protein sythesis by mRNA translation in eucaryotes, which involves unidirectional motion of ribosome complexes along mRNA strands. Theoretical investigations go back to the pioneering work by MacDonald et al. [251], who designed a driven lattice gas model which by now is known as the totally asymmetric exclusion process (TASEP). In this model a single species of particles is hopping unidirectionally and with a uniform rate along a one-dimensional lattice. The only interaction between the particles is hard-core repulsion, which prevents more than one particle from occupying the same site on the lattice; see Fig. 11. Originally intended as a model for an important biological process, this model has by now become one of the paradigms of non-equilibrium physics (for a review see Refs. [252255]).

23 It is impossible to list the multitude of theoretical and experimental work in this area. The reader may consult some of the recent reviews [238-241] and books [236] for a more elaborate discussion and additional references. 


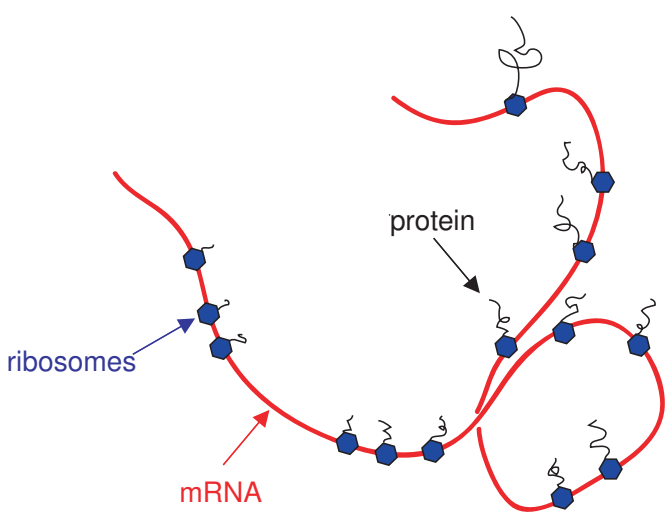

Fig. 10 Illustration of protein synthesis: ribosomes move from codon to codon along a mRNA template, reading off genetic information and thereby generating proteins step by step [256]

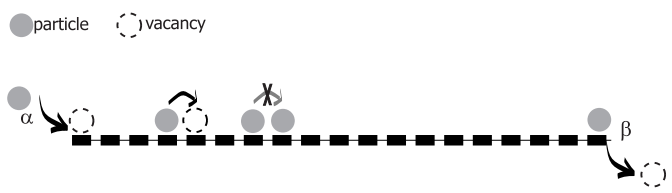

Fig. 11 Illustration of the Totally Asymmetric Simple Exclusion Process with open boundaries. The entrance and exit rates at the left and right end of the one-dimensional lattice are given by $\alpha$ and $\beta$, respectively.

Much of what we know about the dynamics and the steady state of the TASEP and driven lattice gases [60] in general is the result of theoretical work using a broad variety of tools and exploiting the intimate connections between classical and quantum physics, which we eluded to in section 4 . It has been found that the nature of the non-equilibrium steady state of the TASEP depends sensitively on the boundary conditions. For periodic boundary conditions the system reaches a steady state of constant density. Interestingly, density fluctuations are found to spread faster than diffusively [257]. This can be understood by an exact mapping [258] to a growing interface model, whose dynamics in the continuum limit is described in terms of the Kardar-Parisi-Zhang equation [259] and its cousin the noisy Burgers equation [260]. In contrast to such ring systems, open systems with particle reservoirs at the ends exhibit phase transitions upon varying the boundary conditions [261]. This is genuinely different from thermal equilibrium systems where boundary effects usually do not affect the bulk behavior and become negligible if the system is large enough. In addition, general theorems do not even allow equilibrium phase transitions in one-dimensional systems at finite temperatures (if the interactions are not too long-range) [262]. Yet another difference between equilibrium and non-equilibrium processes can be clearly seen on the level of its dynamics. If transition rates between microscopic configurations are obeying detailed balance the system is guaranteed to evolve into thermal equilibrium. Systems lacking detailed balance may still reach a steady state, but at present there are no universal concepts like the Boltzmann-Gibbs ensemble theory for characterizing such non-equilibrium steady states. In most instances one has to resort on solving nothing less than its full dynamics. It is only recently, that exact (non-local) free energy functionals for driven diffusive systems have been derived [263,264].

Recently, driven lattice gas models have found their way back into biological physics. MacDonald's original ideas have been generalized taking into account the finite extension of ribosomes [265, 266]. Also some aspects of intracellular transport, where the molecular motor kinesin moves along microtubules, show close resemblance with the TASEP. As an additional feature one has to account for the fact that microtubules are embedded in a cellular environment with a reservoir of motors in solution. This allows for the motors to attach from the solution to the molecular track and to detach from it to join the reservoir [267, 268]. Typically kinetic rates are such that these motors walk a significant fraction along the molecular track before detaching. It came as quite a surprise that the competition between the non-conservative on/offdynamics and the driven transport along the molecular track leads to a separation between high and low density phases [267]. 


\section{Conclusions and Outlook}

After 100 years many of the central questions surrounding the ubiquitious phenomenon of Brownian motion are still unresolved so that it seems still too early for closing the books and drawing final conclusions. As soon as we leave the caved paths of equilibrium statistical mechanics traced out a century ago, intriguing open problems and promising perspectives abound. Still, we cannot derive the basic assumptions of the founding fathers from the underlying microscopic dynamics, and still we have not managed to generalize reliably the powerful theoretical framework we inherited from them to processes far from equilibrium. We are only starting to appreciate to what extent the processes of life are shaken by fluctuations, just like the pollen grains studied by Brown almost 200 years ago. Life undeniably shows a strong preference for the borderline between perfect order and the erratic dance called Brownian motion, which will, for the foreseeable future, remain a paradigm of the natural sciences in a noisy world.

Acknowledgements We are indepted to Panayotis Benetatos, Matthias Fuchs, and Josef Käs for discussiolns and valuable suggestions. We thank the authors of Refs. [100,219] for permitting the reproduction of Figs. 5 and 8.

\section{References}

[1] R. P. Feynman, R. B. Leighton, and M. L. Sands, The Feynman Lectures on Physics (Addison-Wesley, Reading, 1963), Vol. I.

[2] A. Einstein, Ann. Phys. (Leipzig) 17, 549 (1905).

[3] R. Brown, Edin. New Phil. J. 5, 358 (1828).

[4] A. Brongniart, Ann. Sci. Naturelles 12, 41 (1827).

[5] J. B. Perrin, Discontinuous Structure of Matter, Nobel Lecture, 1926.

[6] L. Bachelier, Annales Scientifiques de l'École Normale Supérieure 17, 21 (1900).

[7] N. Dunbar, Inventing Money: The Story of Long-Term Capital Management and the Legends Behind It (Wiley, Chichester, 2000).

[8] M. von Smoluchowski, Ann. Phys. (Leipzig) 756 (1906).

[9] P. Langevin, Comptes. Rendues 146, 530 (1908).

[10] M. Haw, Physics World (2005), to appear.

[11] P. Coleman, Ann. Henri Poincaré 4, 1 (2003).

[12] N. D. Antunes, L. M. A. Bettencourt, and W. H. Zurek, Phys. Rev. Lett. 82, 2824 (1999).

[13] G. E. Volovik, Phys. Rep. 351, 195 (2001).

[14] P. W. Anderson, Science 177, 393 (1972).

[15] P. M. Chaikin and T. C. Lubensky, Principles of condensed matter physics (Cambridge University Press, Cambridge, 1995).

[16] Soft and Fragile Matter: Nonequilibrium Dynamics, Metastability and Flow, edited by M. E. Cates and M. R. Evans (SUSSP \& IOP, Bristol, 2000).

[17] W. Denk and W. W. Webb, Phys. Rev. Lett. 63, 207 (1989).

[18] P. R. Saulson, Phys. Rev. D 42, 2437 (1990).

[19] B. S. Kerner, The Physics of Traffic. Empirical Freeway Pattern Features, Engineering Applications, and Theory, Springer Series Understanding Complex Systems (Springer, Berlin, 2004).

[20] F. Schweitzer, Brownian Agents and Active Particles. Collective Dynamics in the Natural and Social Sciences, Springer Series in Synergetics (Springer, Berlin, 2003).

[21] D. Challet, A. Chessa, M. Marsili, and Y.-C. Zhang, Quantitative Finance 1, 168 (2001).

[22] R. N. Mantegna and H. E. Stanley, An Introduction to Econophysics: Correlations and Complexity in Finance (Cambridge University Press, Cambridge, 2000).

[23] J.-P. Bouchaud and M. Potters, Theory of Financial Risk and Derivative Pricing: From Statistical Physics to Risk Management, 2nd ed. (Cambridge University Press, Cambridge, 2004).

[24] J. von Neumann and O. Morgenstern, Theory of Games and Economic Behavior (Princeton University Press, Princeton, 1944).

[25] M. Haw, J. Phys.: Condens. Matter 14, 7769 (2002).

[26] S. Chandrasekhar, Rev. Mod. Phys. 15, 1 (1943).

[27] E. Nelson, Dynamical Theories of Brownian Motion (Princeton Univerisity Press, Princeton, 1967), $2^{\text {nd }}$ ed. at http://www.math.princeton.edu/ nelson/books.html. 
[28] C. W. Gardiner, Handbook of Stochastic Methods for Physics, Chemistry and the Natural Sciences, Vol. 13 of Springer Series in Synergetics (Springer, Berlin, 1985).

[29] R. M. Mazo, Brownian Motion. Fluctuations, Dynamics, and Applications (Clarendon, New York, 2002).

[30] N. D. Mermin, Physics Today 57, 10 (2004).

[31] L. Gouy, J. Physique 7, 561 (1888).

[32] C. Wiener, Ann. Phys. 118, 79 (1863).

[33] P. S. Agutter, P. C. Malone, and D. N. Wheatley, J. Hist. Biol. 33, 71 (2000).

[34] A. Fick, Ann. Phys. (Leipzig) 94, 59 (1855).

[35] A. Einstein, Ann. Phys. (Leipzig) 19, 371 (1906).

[36] R. P. Feynman and A. R. Hibbs, Quantum Mechanics and Path Integrals (McGraw-Hill, New York, 1965).

[37] A. Einstein, Ann. Phys. (Leipzig) 22, 569 (1907).

[38] A. Einstein, Ann. Phys. (Leipzig) 33, 1275 (1910).

[39] F. E. Irons, Am. J. Phys. 72, 1059 (2004).

[40] Fluctuation Phenomena, 2 ed., edited by E. W. Montroll and J. L. Lebowitz (Elsevier, Amsterdam, 1987).

[41] R. Kubo, Science 233, 330 (1986).

[42] A. Fölsing, Albert Einstein: a biography (Penguin Books, London, 1997).

[43] J. B. Johnson, Phys. Rev. 32, 97 (1928).

[44] H. Nyquist, Phys. Rev. 32, 110 (1928).

[45] C. Domb, The Critical Point: A historical introduction to the modern theory of critical phenomena (Taylor \& Francis, London, 1996).

[46] P. C. Hohenberg and B. I. Halperin, Rev. Mod. Phys. 49, 435 (1977).

[47] E. Frey and F. Schwabl, Adv. in Phys. 53, 577 (1994).

[48] S. Wiegand, J. Phys.: Condens. Matter 16, R357 (2004).

[49] L. P. Kadanoff and P. C. Martin, Annals of Physics 24, 419 (1963).

[50] D. Forster, Hydrodynamic Fluctuations, Broken Symmetry, and Correlation Functions (Addison Wesley, Redwood City, 1975).

[51] W. Brenig, Statistical Theory of Heat: Nonequilibrium Phenomena (Springer, Heidelberg, 1989).

[52] R. Zwanzig, Nonequilibrium Statistical Mechanics (Oxford University Press, Oxford, 2001).

[53] J. R. Dorfman, An Introduction to Chaos in NonEquilibrium Statistical Mechanics (Cambridge University Press, Cambridge, 1999).

[54] G. Gallavotti, F. Bonetto, and G. Gentile, Aspects of Ergodic, Qualitative and Statistical Theory of Motion (Springer, Berlin, 2004).

[55] L. Bunimovich and Y. G. Sinai, Commun. Math. Phys. 78, 479 (1981).

[56] P. Gaspard, Chaos, Scattering, and Statistical Mechanics (Cambridge University Press, Cambridge, 1998).

[57] F. Bonetto, J. L. Lebowitz, and L. Rey-Bellet, math-ph/0002052, submitted to World Scientific (unpublished).

[58] F. Cecconi, M. Cencini, M. Falcioni, and A. Vulpiani, xx, xx (2005), to appear.

[59] P. Gaspard, M. E. Briggs, M. K. Francis, J. V. Sengers, R. W. Gammon, J. R. Dorfman, and R. V. Calabrese, Nature 394, 865 (1998).

[60] B. Schmittmann and R. Zia, in Phase Transitions and Critical Phenomena, edited by C. Domb and J. Lebowitz (Academic Press, London, 1995), Vol. 17.

[61] L. Berthier and J.-L. Barrat, Phys. Rev. Lett. 89, 095702 (2002).

[62] L. Cipelletti, S. Manley, R. C. Ball, and D. A. Weitz, Phys. Rev. Lett. 84, 2275 (2000).

[63] B. Fabry, G. N. Maksym, J. P. Butler, M. Glogauer, D. Navajas, and J. J. Fredberg, Phys. Rev. Lett. 87, 148102 (2001).

[64] J. Liphardt, S. Dumont, S. B. Smith, I. Tinoco Jr., and C. Bustamente, Science 296, 1832 (2002).

[65] U. Täuber, Critical dynamics, http://www.phys.vt.edu (2003).

[66] C. Jarzynski, Nonequilibrium work theorem for a system strongly coupled to a thermal environment, 2004, cond-mat/0407340.

[67] in Slow relaxations and nonequilibrium dynamics in condensed matter, Vol. Session LXXVII (2002) of Les Houches Summer Schools of Theoretical Physics, edited by J.-L. Barrat, M. Feigelman, J. Kurchan, and J. Dalibard (EDP Sciences \& Springer, Berlin, 2003), pp. 367-521.

[68] P. Sollich, F. Lequeux, P. Hébraud, and M. E. Cates, Phys. Rev. Lett. 78, 2020 (1997).

[69] Spin Glasses and Random Fields, edited by A. P. Young (World Scientific, Singapore, 1997).

[70] M. Buchanan, Nature 425, 556 (2003).

[71] I. E. T. Iben, D. Braunstein, W. Doster, H. Frauenfelder, M. K. Hong, J. B. Johnson, S. Luck, P. Ormos, A. Schulte, P. J. Steinbach, A. H. Xie, and R. D. Young, Physical Review Letters 62, 1916 (1989).

[72] J.-P. Bouchaud and A. Georges, Phys. Rep. 195, 127 (1990). 
[73] J.-P. Bouchaud, L. F. Cugliandolo, J. Kurchan, and M. Mezard, in Spin-glasses and random fields, edited by P. Young (World Scientific, Singapore, 1997), pp. 161-223.

[74] P. G. Debenedetti and F. H. Stillinger, Nature 410, 259 (2001).

[75] A. Crisanti and F. Ritort, J. Phys. A. 36, R181 (2003).

[76] L. F. Cugliandolo and J. Kurchan, Phys. Rev. Lett. 71, 173 (1993).

[77] G. Szamel, Phys. Rev. Lett. 93, 178301 (2004).

[78] D. Gross, Physics World 23 (2004).

[79] G. Gallavotti and E. G. D. Cohen, Phys. Rev. Lett. 74, 2694 (1995).

[80] C. Jarzynski, Phys. Rev. Lett. 78, 2690 (1998).

[81] D. Ruelle, Physics Today 57, 48 (2004).

[82] D. J. Evans and D. J. Searles, Adv. Phys. 51, 1529 (2002).

[83] G. Gallavotti, Foundations of Fluid Dynamics (Springer, Berlin, 2002).

[84] C. Maes and K. Netocný, J. Stat. Phys. 110, 269 (2003).

[85] G. E. Crooks, Phys. Rev. E 61, 2361 (2000).

[86] F. Ritort, Poincaré Seminar 2, 195 (2003).

[87] G. M. Wang, E. M. Sevick, E. Mittag, D. J. Searles, and D. J. Evans, Phys. Rev. Lett. 89, 050601 (2002).

[88] R. van Zon and E. G. D. Cohen, Phys. Rev. Lett. 91, 110601 (2003).

[89] A. Baldassarri, F. Colaiori, and C. Castellano, Phys. Rev. Lett. 90, 060601 (2003).

[90] G. Ehrhardt, S. Majumdar, and A. Bray, Phys. Rev. E 69, 016106 (2004).

[91] W. Feller, An Introduction to Probability Theory and Its Applications (Wiley, London, 1968), Vol. 1.

[92] N. Wiener, Proc. Natl. Acad. Sci. (USA) 7, 253, 294 (1921).

[93] H. Kleinert, Path Integrals in Quantum Mechanics, Statistics, Polymer Physics, and Financial Markets, 3 ed. (World Scientific, Singapore, 2004).

[94] M. Kac, Trans. Am. Math. Soc. 65, 1 (1949).

[95] P. de Gennes, Phys. Lett. A 38, 339 (1972).

[96] M. Doi and S. F. Edwards, The Theory of Polymer Dynamics (Clarendon Press, Oxford, 1986).

[97] J. des Cloizeaux and G. Jannink, Polymers in Solution (Clarendon Press, Oxford, 1990).

[98] Statstical Mechanics of Membranes and Surfaces, Vol. 5 of Jerusalem Winter School for Theoretical Physics, edited by D. Nelson, T. Piran, and S. Weinberg (World Scientific, Singapore, 1989).

[99] L. Schäfer, Excluded Volume Effects in Polymer Solutions as Explained by the Renormalization Group (Springer, Berlin, 1999).

[100] B. Maier and J. Rädler, Phys. Rev. Lett. 82, 1911 (1999).

[101] E. Frey, K. Kroy, J. Wilhelm, and E. Sackmann, in Statistical Mechanics of Semiflexible Polymers: Theory and Experiments, edited by D. Beysens and G. Forgacs (EDP Sciences, Springer Verlag, Berlin 1997), pp. 103.

[102] C. Bustamante, Z. Bryant, and S. Smith, Nature 421, 423 (2003).

[103] M. Peyrard, Nonlinearity 17, R1 (2004).

[104] D. R. Nelson, Statistical Physics of Unzipping DNA, 2004, cond-mat/0309559.

[105] U. Täuber, Adv. Sol. State Phys. 43, 659 (2003).

[106] M. Sasai and P. Wolynes, Proc. Natl. Acad. Sci. (USA) 100, 2374 (2003).

[107] M. Kimura, The neutral theory of molecular evolution (Cambridge University Press, Cambridge, 1983).

[108] R. Fisher, Proc. Roy. Soc. Edinburgh 42, 321 (1922).

[109] A. Griffiths, J. Miller, D. Suzuki, R. Lewontin, and W. Gelbart, An Introduction to Genetic Analysis, 7 ed. (W.H. Freeman, New York, 1999).

[110] T. Liggett, Interacting Particle Systems (Springer Verlag, New York, 1985).

[111] P. Krapivsky and S. Redner, Am. J. Phys. 64, 546 (1996).

[112] D. Ben-Avraham, D. Considine, P. Meakin, S. Redner, and H. Takayasu, J. Phys. A. 23, 4297 (1990).

[113] M. Kimura, Proc. Natl. Acad. Sci. (USA) 41, 144 (1955).

[114] P. Jones and P. Smith, Stochastic Processes: An Introduction (Oxford University Press, New York, 2001).

[115] S. Redner, A Guide to First-Passage Processes (Cambridge University Press, Cambridge, 2001).

[116] N. G. van Kampen, Stochastic Processes in Physics and Chemistry (Elsevier Science B. V., Amsterdam, 1992).

[117] M. Kimura and T. Ohta, Genetics 61, 763 (1969).

[118] H. Kazazian, Science 303, 1626 (2004).

[119] B. Drossel, Adv. Phys. 50, 209 (2001).

[120] E. Baake and W. Gabriel, Ann. Rev. Comp. Phys. 7, 203 (2000).

[121] H. A. Kramers, Physica 7, 284 (1940).

[122] P. Hänggi, P. Talkner, and M. Borkovec, Rev. Mod. Phys. 62, 251 (1990).

[123] M. Delbrück, J. Chem. Phys. 8, 120 (1940).

[124] D. Toussaint and F. Wilczek, J. Chem. Phys. 78, 2642 (1983). 
[125] B. P. Lee, J. Phys. A. 27, 2633 (1994).

[126] H. Hinrichsen, Adv. Phys. 49, 815 (2000).

[127] L. Onsager, Phys. Rev. 37, 405 (1931).

[128] J. Cardy, in Proceedings of mathematical beauty of physics, Vol. 24 of Advanced Series in Mathematical Physics, edited by J.-B. Zuber (World Scientific, Singapore, 1997), p. 113, cond-mat/9607163.

[129] P. Guptasarma, Bioessays 17, 987 (1995).

[130] J. L. Spudich and D. E. Koshland, Nature 262, 467 (1976).

[131] H. McAdams and A. Arkin, Proc. Natl. Acad. Sci. (USA) 94, 814 (1997).

[132] M. Elowitz, A. Levine, E. Siggia, and P. Swain, Science 297, 1183 (2002).

[133] H. McAdams and A. Arkin, Trends Genet. 15, 65 (1999).

[134] V. Henri, C. R. Acad. Sci., Paris 146, 1024 (1908).

[135] F. Amblard, A. C. Maggs, B. Yurke, A. N. Pargellis, and S. Leibler, Phys. Rev. Lett. 77, 4470 (1996).

[136] F. Gittes, B. Schnurr, P. D. Olmsted, F. C. MacKintosh, and C. F. Schmidt, Phys. Rev. Lett. 79, 3286 (1997).

[137] J. C. Crocker, M. T. Valentine, E. R. Weeks, T. Gisler, P. D. Kaplan, A. G. Yodh, and D. A. Weitz, Phys. Rev. Lett. 85, 888 (2000).

[138] M. B. Forstner, D. S. Martin, A. M. Navar, and J. A. Käs, Langmuir 19, 4876 (2003).

[139] G. Maret and P. E. Wolf, Z. Phys. B: Condens. Matter 65, 409 (1987).

[140] D. A. Weitz and D. J. Pine, in Dynamic Light Scattering, edited by W. Brown (Oxford University Press, New York, 1993), Chap. 16, pp. 652-720.

[141] J. M. Schurr, Biophys. J. 79, 1692 (2000).

[142] Fluorescence Correlation Spectroscopy. Theory and Applications, Vol. 65 of Springer Series in Chemical Physics, edited by R. Rigler and E. S. Elson (Springer, Heidelberg, 2001).

[143] A. R. Bausch, W. Möller, and E. Sackmann, Biophys. J. 76, 573 (1999).

[144] A. Heilbronn, Jahrb. Wiss. Bot. 61, 284 (1922).

[145] M. F. Shlesinger, G. M. Zaslavsky, and J. Klafter, Nature 363, 31 (1993).

[146] B. Mandelbrot, The Fractal Geometry of Nature (Freeman, San Francisco, 1983).

[147] C. Tsallis, S. V. F. Levy, A. M. C. Souza, and R. Maynard, Physical Review Letters 75, 3589 (1995).

[148] R. Metzler and J. Klafter, Phys. Rep. 339, 1 (2000).

[149] M. Weiss, M. Elsner, F. Kartberg, and T. Nilsson, Biophys. J. 87, 3518 (2004).

[150] I. M. Tolić-Nørrelykke, E.-L. Munteanu, G. Thon, L. Oddershede, and K. Berg-Sørensen, Phys. Rev. Lett. 93, 078102 (2004).

[151] A. Ott, J. Bouchaud, D. Langevin, and W. Urbach, Phys. Rev. Lett. 65, 2201 (1990).

[152] I. Y. Wong, M. L. Gardel, D. R. Reichman, E. R. Weeks, M. T. Valentine, A. R. Bausch, and D. A. Weitz, Phys. Rev. Lett. 92, 178101 (2004).

[153] E. Ben-Naim, S. Redner, and D. ben Avraham, Phys. Rev. A 45, 7207 (1992).

[154] D. ben Avraham and S. Havlin, Diffusion and Reaction in Fractals (Cambridge University Press, Cambridge, 2000).

[155] D. Stauffer and A. Aharony, Introduction to Percolation Theory, 2 ed. (Taylor and Francis, London, 1994).

[156] C. Tzanakis and A. Grecos, Transport Theor. Stat. Phys. 28, 325 (1999).

[157] J. A. McLennan, Introduction to Non-Equilibrium Statistical Mechanics (Prentice-Hall, New York, 1988).

[158] J. R. Dorfman and E. G. D. Cohen, Phys. Lett. 16, 124 (1965).

[159] B. J. Alder and T. E. Wainwright, Phys. Rev. Lett. 18, 988 (1967).

[160] M. H. Ernst, E. H. Hauge, and J. M. J. van Leeuwen, Phys. Rev. Lett. 25, 1254 (1970).

[161] T. Keyes and A. J. Masters, Adv. Chem. Phys. 58, 1 (1985).

[162] L. D. Landau and E. M. Lifshitz, Fluid Mechanics, Vol. 6 of Course of Theoretical Physics (Pergamon Press, London, 1963).

[163] D. J. Evans and G. P. Morriss, Statistical Mechanics of NonEquilibrium Liquids (Academic Press, London, 1990).

[164] M. Fuchs and K. Kroy, J. Phys.: Condens. Matter 14, 9223 (2002).

[165] P. G. Wolynes, Phys. Rev. A 13, 1235 (1976).

[166] L. Bocquet and J.-L. Barrat, Phys. Rev. E 49, 3079 (1994).

[167] M. H. J. Hagen, I. Pagonabarraga, C. P. Lowe, and D. Frenkel, Physical Review Letters 78, 3785 (1997).

[168] M. L. Gardel, M. T. Valentine, J. C. Crocker, A. R. Bausch, and D. A. Weitz, Phys. Rev. Lett. 91, 158302 (2003).

[169] C. Lutz, M. Kollmann, and C. Bechinger, Phys. Rev. Lett. 93, 026001 (2004).

[170] P. N. Pusey, in Liquids, Freezing and Glass Transition, edited by J. P. Hansen, D. Levesque, and J. Zinn-Justin (Elsevier, North Holland, Amsterdam, 1991), p. 763. 
[171] W. B. Russel, D. A. Saville, and W. R. Schowalter, Colloidal Dispersions (Cambridge University Press, Cambridge, 1991).

[172] D. Frenkel, in Soft and Fragile Matter: Nonequilibrium Dynamics, Metastability and Flow, edited by M. E. Cates and M. R. Evans (SUSSP \& IOP, Bristol, 2000), pp. 113-144.

[173] J. P. Hansen and I. R. McDonald, Theory of simple liquids, 2 ed. (Academic Press, London, 1986).

[174] J. Herzfeld, Acc. Chem. Res. 29, 31 (1996).

[175] P. Chaikin, in Soft and Fragile Matter: Nonequilibrium Dynamics, Metastability and Flow, edited by M. E. Cates and M. R. Evans (SUSSP \& IOP, Bristol, 2000), pp. 315-348.

[176] B. J. Alder and T. E. Wainwright, J. Chem. Phys. 27, 1208 (1957).

[177] W. G. Hoover and F. H. Ree, J. Chem. Phys. 49, 3609 (1968).

[178] P. N. Pusey and W. van Megen, Nature 320, 340 (1986).

[179] J. Zhu, M. Li, R. Rogers, W. Meyer, R. H. Ottewill, S. Space Shuttle Crew, Russel, and C. P. M., Nature 387, 883 (1997).

[180] B. Cipra, Science 281, 1267 (1998).

[181] T. M. Bergfors, Protein Crystallization (International University Line, La Jolla, CA, 1999).

[182] W. C. K. Poon, Phys. Rev. E 55, 3762 (1997).

[183] A. Einstein, Ann. Phys. (Leipzig) 17, 132 (1905).

[184] S. John, Physics Today 44, 32 (1991).

[185] W. Götze, in Liquids, Freezing and Glass Transition, edited by J. P. Hansen, D. Levesque, and J. Zinn-Justin (Elsevier, North Holland, Amsterdam, 1991), p. 287.

[186] M. Fuchs, J. Non-Cryst. Solids 172-174, 241 (1994).

[187] W. Götze and L. Sjögren, Rep. Prog. Phys. 55, 241 (1992).

[188] W. Götze, J. Phys.: Condens. Matter 11, A1 (1999).

[189] W. van Megen and S. M. Underwood, Phys. Rev. Lett. 70, 2766 (1993).

[190] W. Kob, J. Phys.: Condens. Matter 11, R85 (1999).

[191] M. E. Cates, Ann. Henri Poincaré 4, S647 (2003).

[192] J.-P. Bouchaud, L. Culiandolo, J. Kurchan, and M. Mezard, Physica A 226, 243 (1996).

[193] K. Kawasaki and K. Fuchizaki, J. Non-Cryst. Solids 235-237, 57 (1998).

[194] K. N. Pham, A. M. Puertas, J. Bergenholtz, S. U. Egelhaaf, A. Moussaid, P. N. Pusey, A. B. Schofield, M. E. Cates, M. Fuchs, and W. C. K. Poon, Science 296, 104 (2002).

[195] F. Sciortino, Nature Mat. 1, 145 (2002).

[196] F. Brochard and J. F. Lennon, J. Phys. (Paris) 36, 1035 (1975).

[197] W. Helfrich and W. Harbich, Chem. Scr. 25, 32 (1985).

[198] F. MacKintosh, J. Käs, and P. Janmey, Phys. Rev. Lett. 75, 4425 (1995).

[199] B. Hinner, M. Tempel, E. Sackmann, K. Kroy, and E. Frey, Phys. Rev. Lett. 81, 2614 (1998).

[200] T. Halpin-Healy and Y.-C. Zhang, Phys. Rep. 254, 215 (1995).

[201] M. Kardar, Phys. Rep. 301, 85 (1998).

[202] D. Fisher, Phys. Rep. 301, 113 (1998).

[203] D. Panja, Phys. Rep. 393, 87 (2004).

[204] P. G. de Gennes, Scaling Concepts in Polymer Physics (Cornell University Press, Ithaca and London, 1979).

[205] S. A. Safran, Statistical Thermodynamics of Surfaces, Interfaces, and Membranes (Westview Press, Boulder, 2003).

[206] U. Seifert, Adv. Phys. 46, 13 (1997).

[207] R. Lipowsky, Nature 349, 475 (1991).

[208] Structure and Dynamics of Membranes: From Cells to Vesicles. Handbook of Biological Physics, Vol. 1., edited by R. Lipowsky and E. Sackmann (Elsevier Science, Amsterdam, 1995).

[209] D. Nelson, Defects \& Geometry in Condensed Matter Physics, 1 ed. (Cambridge University Press, Cambridge, 2002).

[210] G. Blatter, M. Feigelman, V. Geshkenbein, A. Larkin, and V. Vinokur, Rev. Mod. Phys. 66, 1125 (1994).

[211] E. Frey and D. Nelson, J. Phys. I (France) 1, 1715 (1991).

[212] F. Gittes, B. Mickey, J. Nettleton, and J. Howard, Journal of Cell Biology 120, 923 (1993).

[213] A. Caspi, M. Elbaum, R. Granek, A. Lachish, and D. Zbaida, Phys. Rev. Lett. 80, 1106 (1998).

[214] L. Le Goff, O. Hallatschek, E. Frey, and F. Amblard, Phys. Rev. Lett. 89, 258101 (2002).

[215] M. Hohenadl, T. Storz, H. Kirpal, K. Kroy, and R. Merkel, Biophys. J. 77, 2199 (1999).

[216] O. Hallatschek, E. Frey, and K. Kroy, to be published (unpublished).

[217] K. J. Wiese, in Polymerized membranes, a review, Vol. 19 of Phase Transitions and Critical Phenomena, edited by C. Domb (Academic Press, Oxford, 2000), Chap. 2.

[218] T. C. B. McLeish, Adv. Phys. 51, 1379 (2002). 
[219] J. Käs, H. Strey, and E. Sackmann, Nature 368, 226 (1994).

[220] D. E. Smith, T. T. Perkins, and S. Chu, Phys. Rev. Lett. 75, 4146 (1995).

[221] S. H. Chong and M. Fuchs, Phys. Rev. Lett. 88, 185702 (2002).

[222] R. Everaers, S. K. Sukumaran, G. S. Grest, C. Svaneborg, A. Sivasubramanian, and K. Kremer, Science 303, 823 (2004).

[223] J. P. Wittmer, H. Meyer, J. Baschnagel, A. Johner, S. Obukhov, L. Mattioni, M. Mller, and A. N. Semenov, Phys. Rev. Lett. 93, 147801 (2004).

[224] S. Ramaswamy, J. Toner, and J. Prost, Phys. Rev. Lett. 84, 3494 (2000).

[225] D. L. J.-B. Manneville, P. Basserau and J. Prost, Phys. Rev. Lett. 82, 4356 (1999).

[226] F. J. Nedelec, T. Surrey, A. C. Maggs, and S. Leibler, Nature 389, 305 (1997).

[227] D. Humphrey, C. Duggan, D. Saha, D. Smith, and J. Käs, Nature 416, 413 (2002).

[228] J. Uhde, M. Keller, E. Sackmann, A. Parmeggiani, and E. Frey, Phys. Rev. Lett. (in press), (2004).

[229] K. Kruse, J. F. Joanny, F. Jlicher, J. Prost, and K. Sekimoto, Phys. Rev. Lett. 92, 078101 (2004).

[230] P. Hänggi, P. Talkner, and m. Borkovec, Rev. Mod. Phys. 62, 251 (1990).

[231] Y. Hatwalne, S. Ramaswamy, M. Rao, and R. A. Simha, Phys. Rev. Lett. 92, 118101 (2004).

[232] H. v. Helmholtz, in Ostwalds Klassiker der Exakten Wissenschaften (Harri Deutsch, Thun, 1996), Vol. 1.

[233] A. Huxley, Prog. Biophys. 7, 255 (1957).

[234] T. Hill, Prog. Biophys. Mol. Biol. 28, 267 (1974).

[235] A. D. Mehta, M. Rief, J. A. Spudich, D. A. Smith, and R. M. Simmons, Science 283, 1689 (1999).

[236] J. Howard, Mechanics of Motor Proteins and the Cytoskeleton (Sinauer Associates, Sunderland, MA, 2001).

[237] P. Hänggi and R. Bartussek, Lect. Notes Phys. 476, 294 (1996).

[238] R. Astumian, Science 276, 917 (1997); R. Astumian and P. Hänggi, Physics Today 55, 33 (2002).

[239] F. Jülicher, A. Ajdari, and J. Prost, Rev. Mod. Phys. 69, 1269 (1997).

[240] P. Reimann, Phys. Rep. 361, 57 (2002).

[241] A. Parmeggiani and C. Schmidt, in Functions and Regulation of Cellular Systems: Experiments and Models, edited by A. Deutsch, J. Howard, M. Falcke, and W. Zimmermann (Birkhäuser, Basel, 2004), p. 151.

[242] P. Hänggi, F. Marchesoni, and F. Nori, Annalen der Physik 14, xxxx (2005); cond-mat/0410033.

[243] A. Bug and B. Berne, Phys. Rev. Lett. 59, 948 (1987).

[244] A. Ajdari and J. Prost, C.R. Acad. Sci. Paris II 315, 1635 (1992).

[245] R. D. Astumian and M. Bier, Phys. Rev. Lett. 72, 1766 (1994).

[246] M. von Smoluchowski, Phys. Zeitschr. 13, 1069 (1912).

[247] J. Rousselet, L. Salome, A. Ajdari, and J. Prost, Nature 370, 446 (1994).

[248] L. Faucheux, L. Bourdieu, P. Kaplan, and A. Libchaber, Phys. Rev. Lett. 74, 1504 (1995).

[249] M. Sarikay, C. Tamaeler, A. Jen, K. Schulten, and F. Baneyx, Nature Materials 2, 577 (2003).

[250] K. Svoboda, P. P. Mitra, and S. M. Block, Proc. Natl. Acad. Sci. (USA) 91, 11782 (1994).

[251] C. MacDonald, J. Gibbs, and A. Pipkin, Biopolymers 6, 1 (1968).

[252] H. Spohn, Large Scale Dynamics of Interacting Particles (Springer Verlag, New York, 1991).

[253] B. Derrida and M. Evans, in Nonequilibrium Statistical Mechanics in One Dimension, edited by V. Privman (Cambridge University Press, Cambridge, 1997), Chap. 14, pp. 277-304.

[254] D. Mukamel, in Soft and Fragile Matter, edited by M. Cates and M. Evans (Institute of Physics Publishing, Bristol, 2000), pp. 237-258.

[255] G. Schütz, in Phase Transitions and Critical Phenomena, edited by C. Domb and J. Lebowitz (Academic Press, San Diego, 2001), Vol. 19, pp. 3-251.

[256] B. Alberts, D. Bray, J. Lewis, M. Raff, K. Roberts, and J. D. Watson, Molecular Biology of the cell, $3^{\text {rd }}$ ed. (Garland Publ., New York, 1994).

[257] H. van Beijeren, R. Kutner, and H. Spohn, Phys. Rev. Lett. 54, 2026 (1985).

[258] P. Meakin, P. Ramanlal, L. Sander, and R. Ball, Phys. Rev. A 34, 5091 (1986).

[259] M. Kardar, G. Parisi, and Y.-C. Zhang, Phys. Rev. Lett. 56, 889 (1986).

[260] D. Forster, D. R. Nelson, and M. J. Stephen, Phys. Rev. A 16, 732 (1977).

[261] J. Krug, Phys. Rev. Lett. 67, 1882 (1991).

[262] L. Landau and E. Lifshitz, Statistical Physics I (Pergamon Press, New York, 1980).

[263] B. Derrida, J. Lebowitz, and E. Speer, Phys. Rev. Lett. 87, 150601 (2001).

[264] B. Derrida, J. Lebowitz, and E. Speer, Phys. Rev. Lett. 89, 030601 (2002).

[265] L. Shaw, R. Zia, and K. Lee, Phys. Rev. E 68, 021910 (2003).

[266] T. Chou, 85, 755 (2003).

[267] A. Parmeggiani, T. Franosch, and E. Frey, Phys. Rev. Lett. 90, 068810 (2003).

[268] S. Klumpp and R. Lipowsky, J. Stat. Phys. 113, 233 (2003). 